\title{
Keck versus Wepp: Ein Erb- und Ehegutsprozess in Wien zu Beginn der Rezeptionszeit
}

\section{Widmung}

Der folgende Beitrag wurde inspiriert durch Fragmente von zwei umfangreichen, in den Quellen zur Geschichte der Stadt Wien auszugsweise publizierten Gerichtsurkunden ${ }^{1}$, anhand welcher Wilhelm Brauneder über Jahrzehnte hinweg in seinen Übungen an der Wiener Juristenfakultät Generationen von Anfängern des rechtswissenschaftlichen Studiums beispielhaft darlegte, in welchen Formen sich die Rezeption des (römisch-)gemeinen Rechts zu Beginn der Frühneuzeit im heimischen Rechtsleben geltend machte. ${ }^{2}$ Die erwähnten Gerichtsurkunden geben den Verlauf eines Prozesses vor dem Wiener Stadtgericht, dem Rat, wieder, der sich in mehreren Phasen mit längeren Unterbrechungen von Jahresbeginn 1507 bis September 1516 erstreckt. Im Mittelpunkt dieses Rechtsstreits steht der Nachlass des im Herbst 1506 verstorbenen Wiener Stadtschreibers Dr. Stefan Vorchtenauer ${ }^{3}$, der zum Teil von dessen Witwe Margarethe, und zum Teil vom Stiefvater des Erblassers, dem damaligen Wiener Bürgermeis-

\footnotetext{
* Siehe unten IV.B (Anm. 103).

${ }^{1}$ LAMPL, Haupturkunden), Nr. 5852 (198-213) und Nr. 6112 (371-392).

${ }^{2}$ Vgl. BRAUnEDER, Privatrechtsgeschichte, Quellen Nr. 18 und 19. Bereits 2011 wurde bei UTB/Böhlau (Wien) eine Umarbeitung des Skriptums als Studienbuch unter dem Titel Europäische Privatrechtsgeschichte angekündigt.

${ }^{3}$ Dazu genauer unten IV.A.
}

ter Paul Keck ${ }^{4}$, in Besitz genommen worden ist. In dem Verfahren spielt unter anderem auch die Frage der Anerkennung von stillschweigenden Pfandrechten des (römisch-)gemeinen Rechts in der Praxis der heimischen Gerichte am Übergang vom Mittelalter zur Frühneuzeit eine wichtige Rolle. Wilhelm Brauneder setzte sich damit bei seinem „wissenschaftlichen Debüt", am 11. Österreichischen Historikertag in Innsbruck 1971, auseinander und nahm dabei auch auf ein paar Splitter des im Folgenden skizzierten Prozesses $^{5}$ Bezug. Vier Jahrzehnte später gehört er nun - seit Oktober 2011 - dem Stand der Emeriti an: Aus diesem Anlass sei ihm, verbunden mit allen guten Wünschen, der folgende Beitrag gewidmet. $^{6}$

\footnotetext{
${ }^{4}$ PERGER, Ratsbürger 134ff.: Bürgermeister 1504-1508. ${ }^{5}$ BRAUNEDER, Stillschweigenden Hypotheken 227. Vgl. auch BRAUNEDER, Juristen 33.

${ }^{6}$ Die folgende Darstellung konzentriert sich auf die erste Phase des Rechtsstreits, welche in das Jahr 1507 fällt (II.E); die zweite, 1509 bis 1516 anschließende Phase wird in einem Ausblick bloß kursorisch gestreift (III.). Ein Anhang (IV.B) enthält die Edition des Inventars über das von Stadtschreiber Vorchtenauer hinterlassene Vermögen, darunter unter anderem eine ansehnliche juristische Fachbibliothek.
} 


\section{Sachverhalt und Verlauf des Verfahrens im Jahr 1507}

\section{A. Anlass des Rechtsstreits}

Am 8. Oktober 1506 erscheint vor dem Rat der Stadt Wien Bürgermeister Paul Keck und legt das versiegelte Geschäft ${ }^{7}$ des - wohl knapp davor - verstorbenen ${ }^{8}$ Wiener Stadtschreibers Dr. Stefan Vorchtenauer vor. ${ }^{9}$ Außen hat der Testator eigenhändig den Vermerk angebracht, dass sich darin sein "letster entlicher Will" befinde, dass er diesen eigenhändig verfasst und mit seinem Siegel ${ }^{10}$ verschlossen habe. Als Einbringer des Geschäfts im Rat fungiert sein Stiefvater Bürgermeister Keck, er ist als Willensvollstrecker ${ }^{11}$ eingesetzt worden und als solcher ermächtigt (mit "ganzen gewalt und macht"), das Geschäft zu öffnen (,aufzubrechen“) und dem Rat vorzulegen („,zu weisen“). Nachfolger seines gesamten Vermögens sind $\mathrm{zu}$ gleichen Teilen (,ainem als vil als dem andern“) die Ehefrau („Hausfraw") Margarethe (Rietenberger) und der gemeinsame Sohn Egidius. Von Bestimmungen über die Verwaltung des Anteils seines minderjährigen Sohns und die Entlohnung der Dienst-

${ }^{7}$ LAMPL, Haupturkunden, Nr. 5836a, datiert mit 23. 8. 1506; vgl. auch LAMPL, Haupturkunden, Nr. 5844. Zum Institut des Geschäfts im Wiener Rechtsleben des Spätmittelalters: BRAUNEDER, JARITZ, Stadtbücher 1, 17f., sowie jüngst NESCHWARA, Rechtsformen letztwilliger Verfügungen $135 \mathrm{f}$.

${ }^{8}$ Vorchtenauer weist in seiner Verfügung (s.u. Anm. 13) darauf hin, dass er erkrankt gewesen ist.

${ }^{9}$ Nach Einbringen und Veröffentlichung wird das Geschäft in das "Statbuch zu Wienn geschriben", von "Gabriel Guet-Rater, Licentiat [der Rechte], Statschreiber $z u$ Wienn". Von diesem (sog. kleinen) Wiener Stadtbuch sind nur noch Reste der Eintragungen für das Jahr 1395 sowie für den anschließenden Zeitraum von 1396-1430 erhalten. Siehe dazu BRAUNEDER, JARITZ, Stadtbücher 1, 9.

${ }^{10} \mathrm{Vgl}$. die Siegelbeschreibung in LAMPL, Haupturkunden, Nr. 5811; ferner die Abbildung bei UHLIRZ, Quellen 188.

${ }^{11}$ Dazu NeSchWARA, Willensvollstrecker $12 \mathrm{f}$. leute $^{12}$ sowie unbedeutenden Vermächtnissen ${ }^{13}$ abgesehen, enthält das Geschäft auch eine Anordnung über die Haftung der Erben für die vom Erblasser nachgelassenen Schulden, sie sollen aus dem Nachlassvermögen bezahlt werden. ${ }^{14}$ Besonderes Augenmerk schenkt der Erblasser dabei Geldern, welche er noch "von Herrn Lucasen seligen Kinder wegen in die Gerhabschaft schuldig" ist. Vorchtenauer hat diese Gelder nach eigener Angabe für sich und seine Ehefrau „angriffen und in [...] baider Nutz gekhert", sich also offenbar zu eigenmächtiger Nutzung angeeignet. Er ersucht seine Ehefrau, sie möge ihm „zue Ern" diese Schulden „bezalen auf das beldist", weil "sy hat des als wol genossen", also dieselben Vorteile daraus gezogen habe, wie er selbst. Als Willensvollstrecker (,zu Ausrichter des Gescheffts") bestimmt er neben seinem Stiefvater auch seine Ehefrau Margarethe, welche von ihm aber angewiesen wird, sich dabei von ihrem Schwiegervater anleiten zu lassen (,daz sy das alles handl mit Rat und Wissen [...] meines lieben Herren und Vatern Pauln Kekhen").

Hierbei ist es aber zu keinem Einvernehmen gekommen. Margarethe Rietenberger ist wegen einer schweren Erkrankung von der Publizierung des Geschäftes zunächst nicht in Kenntnis gesetzt worden ${ }^{15}$, und ihr Schwiegervater, Bürgermeister Keck, hat inzwischen - vermutlich bald nach dem Ableben seines Stiefsohns -

\footnotetext{
12 „Darnach ist mein will, daz mein Hausfraw all mein Dienstvolkh erberlich abfertig und bezal."

13 "Mer schaff ich Hannsen Schundorffer, Mauter underm Schotenthor, mein füchseine Häsugkhen, die mit rotem Samt verbraembt ist, darumb daz er mir in meiner Krankhait aufgewart hat. Item Johannsen Grymmen schaff ich meinen täglichen Rockh, der mit weissem Tuch underzogen und mit schwarzem Sammat verbraembt ist, und darzu mein praune atlaseine Joppen."

$14,[$,... dann sy hat noch wol sovil das ich ir laß, davon sy das obbemelt bezalen mag."

${ }^{15}$ Vgl. unten II. D.2. und Anm. 50
} 
durch Stadtrichter Sigmund Pernfußs ${ }^{16}$ die "Sperre" des im Rathaus befindlichen Nachlasses von Vorchtenauer vornehmen lassen. ${ }^{17} \mathrm{Zu}$ einem späteren Zeitpunkt, vermutlich zu Jahresende 1506 oder mit Jahresbeginn 1507, wird auf Anordnung des Bürgermeisters ein Teil der Verlassenschaft separiert, nämlich das in Verwahrung von Vorchtenauer als Willensvollstrecker nach Lukas Schnitzer, dem "Ungelter" (Steuereinnehmer) von Klosterneuburg, verbliebene Vermögen von dessen minderjährigen Kindern. Es ist in mehreren Truhen und "Laden“ in das "Bürgermeisterhaus" verbracht worden. Wegen des Vorwurfs der Witwe von Vorchtenauer, "dass dabei etwas abhanden gekommen" sei, werden auf Veranlassung der beiden mit der Verbringung dieses Vermögens beauftragten städtischen Amtsleute, Ratsdiener Phennelannder und Schrannenschreiber Pheyl, am 15./16. Jänner 1507 vor öffentlichem Notar ${ }^{18}$ die bei diesem Vorgang anwesenden Zeugen ${ }^{1920}$, und am 16. Jänner auch die Witwe von Vorchtenauer einvernommen. Sie befindet sich zu diesem Zeitpunkt infolge ihrer Erkrankung nicht "in irer Behausung “21, sondern im Rathaus und wird über die von ihr ausgestreuten Vorwürfe, sie sei nicht davon in Kenntnis gesetzt worden, dass "Herrn Lucasen Gutt austragen solt werden", und habe sich dieses Vorgehen "on Behelf aller Rechten" gefallen habe lassen müssen, befragt. Nachdem ihr von Ratsdiener Phennelannder vorgehalten wird, "er hab ir solhs khunt

\footnotetext{
${ }^{16}$ Er was Ratsherr von 1497-1499 (in diesem Zeitraum auch Stadtkämmerer) sowie im Jahr 1509 sowie von 1503-1508 Stadtrichter. Dazu PERGER, Ratsbürger 169.

${ }^{17}$ LAMPL, Haupturkunden, Nr. 5844. Zum Terminus „Sperre“ BRAUnEDER, JARITZ, Stadtbücher 1, $15 \mathrm{f}$.

${ }^{18}$ NESCHWARA, Notariat.

${ }^{19}$ Zwei Wiener Stadtbürger sowie ein Knabe namens "Thomandl", Thomas Swanntzer, der Kanzleigehilfe von Stadtschreiber Vorchtenauer, dem - offenbar auf Veranlassung der Witwe von Vorchtenauer - zwei wertvolle Gläser aus einer Truhe überlassen werden. ${ }^{20}$ LAMPL, Haupturkunden, Nr. 5844 (192f.).

${ }^{21}$ Dazu auch unten Anm. 47.
}

gethan, [und von ihr] auch die Schlussel [...] zu dem Gwelb“ im Rathaus, wo das Nachlassvermögen Vorchtenauers verwahrt wurde „zu geben begert", muss sie ihre Behauptung abschwächen. Sie gibt nun zu, zwar in Kenntnis über die Separation von Vermögen ihres früheren Mannes gewesen zu sein, beteuert aber weiterhin, es sei ihr nicht bewusst gewesen, dass es sich um das Vermögen von Lukas Schnitzers hinterlassenen Kindern gehandelt habe.

\section{B. Beginn des Rechtsstreits}

Im Anschluss an ihre Einvernahme vom 16. Jänner wird der im Rathaus von den städtischen Amtsleuten unversperrt zurückgelassene Rest von Vorchtenauers Nachlass durch seine Witwe Margarethe in Besitz genommen. Nach diesem Zeitpunkt, jedenfalls aber noch vor Anfang März 1507, hat sie sich wieder verehelicht, und zwar mit dem Anwalt Dr. Johann Wepp.22 Dies ist offenbar der Zeitpunkt, ab dem sich ihr Verhältnis zum Stiefvater ihres früheren Mannes verschlechtert hat: Paul Keck klagt nämlich nun gegen seine ehemalige Schwiegertochter auf Bezahlung dessen, was sein Stiefsohn Vorchtenauer "von Herrn Lucasen seligen Kinder wegen in die Gerhabschaft schuldig“" geblieben ist. Paul Keck führt die Klage nicht in amtlicher Eigenschaft als Bürgermeister, sondern als Vormund seiner Enkelin ${ }^{23}$ Maria, der minderjährigen Tochter des Lukas Schnitzers. Der Klage schließt sich auch dessen Witwe Barbara an. Sie ist die Tochter von Bürgermeister Keck und die Mutter von Maria und hat sich inzwischen ebenfalls wieder verehelicht, und zwar mit Hanns Hartl aus Krems. ${ }^{24}$

\footnotetext{
${ }^{22}$ Er ist an der Universität Wien (allgemeine Matrikel sowie in den Akten der Theologischen und Artistischen Fakultät) nicht nachweisbar.

${ }^{23}$ Dass Paul Keck Marias Großvater väterlicherseits ist, geht aus der Gerichtsurkunde an späterer Stelle (siehe unten II.D.1.) hervor; siehe auch LAMPL, Haupturkunden, Nr. 5852 (208).

${ }^{24}$ LAMPL, Haupturkunden, Nr. 5852 (198).
} 
Im Prozess wird sie von Martin Keck, dem Sohn des Bürgermeisters, vertreten.

Die Konstellation auf Kläger- und Beklagtenseite zeigt klar, dass es sich um einen familieninternen Streit zwischen der Witwe des Erblassers und dessen Verwandten (Stiefvater, Schwägerin und Nichte) handelt. Dem zeitgenössischen Verfahrensrecht ${ }^{25}$ entsprechend handeln anstelle der Streitteile sogenannte Vorsprecher:26 Die Beklagte Margarethe wird von ihrem nunmehrigen Ehemann Dr. Johann Wepp ${ }^{27}$, einem professionellen, wohl im römischen Recht ausgebildeten Juristen, vertreten. Auf Klägerseite agieren mit Paul Keck und seinem Sohn Martin zwei im Rechtsleben erfahrene städtische Funktionäre:28 Paul Keck ist als Vormund der Maria Schnitzer deren gesetzlicher Vertreter und damit zur Prozessvertretung berufen. Martin Keck ist zwar akademisch gebildet ${ }^{29}$, aber kein graduierter Jurist, er fungiert als rechtsgeschäftlich Bevollmächtigter (mit „brieflichen Gewalt") der Barbara Schnitzer. Die Rolle der Vorsprecher als Anwälte der Parteien und damit als Wortführer im Prozess spiegelt sich im Wortlaut der über den Verlauf des Verfahrens aufgenommenen Gerichtsurkunde allerdings nicht konsequent wider. $^{30}$

${ }^{25}$ Dazu WeSENER, Einflüsse und Geltung 56ff. mit Literaturhinweisen.

${ }^{26}$ NESCHWARA, Anwalt; WrABETZ, Rechtsanwälte 17f., besonders 18; vgl. BUCHDA, CORDES, Anwalt.

${ }^{27}$ Er ist an der Universität Wien nicht nachweisbar.

${ }^{28}$ Beide entstammen einer Ratsherrenfamilie, Paul Keck (aus Röschitz/NÖ, daher auch Röschitzer; † um 1509/13) ist als privilegierter Kaufmann (Laubenherr) 1485, 1490, 1494/96, 1500, 1503 und 1509 Ratsherr, 1495 auch Grundbuchsverwalter, 1485/88 auch Steuerherr, sowie 1490/93, 1497/99 und 1504/8 Bürgermeister; Martin Keck († um 1524/33) ist 1512/16 und 1519/21 Ratsherr, 1519/20 auch Grundbuchsverwalter und 1520 Steuerherr: PERGER, Ratsbürger 176f.

${ }^{29}$ SZAIVERT, GALL, Matrikel 195 (1487 II A 36).

${ }^{30}$ LAMPL, Haupturkunden, Nr. 5852. Auf Seiten der Kläger findet in der Gerichtsurkunde ausschließlich Paul Keck Erwähnung. In einem späteren Stadium des Prozesses, nach seinem Tod (1514), kommt es zu
Der Prozess $^{31}$ wird am 13. März 1507 eröffnet $^{32}$, er zieht sich nach Vorbringen von Klagsbegehren sowie -beantwortung und dreimaligem Wechsel der Schriftsätze bis Mitte August 1507.33 Den Vorsitz im Rat ${ }^{34}$ führt nicht wie sonst im Allgemeinen der Bürgermeister ${ }^{35}$, weil er als Vormund und Vorsprecher auf Klägerseite auftritt und daher von der Prozessleitung ausge-

einem Wechsel auf Klägerseite. An die Stelle von Paul Keck tritt nun sein Sohn Dionys (zu ihm unten Anm. 71) offenbar als Vormund der Maria Schnitzer). Die Gerichtsurkunde spricht nun aber ständig in der Mehrzahl von "Advocaten" auf Seiten des Klägers, ohne diese aber namentlich anzuführen: LAMPL, Haupturkunden, Nr. 6112 (hier ab 381 passim). Auf der Beklagtenseite wird in der Gerichtsurkunde in der ersten Phase nahezu ausschließlich Dr. Wepp, in der zweiten dagegen meist die Beklagte als Wortführerin angegeben.

31 VOLTELINI, Rezeption 88f.

${ }^{32}$ LAMPL, Haupturkunden, Nr. 5852.

${ }^{33}$ Gerichtsurkunde vom 12. 8. 1507: ebd. 198, 204, 213

(„,beschehen an phinztag nach sand Laurentzen Tag“).

${ }^{34}$ Der Rat ist im Zivilverfahren grundsätzlich erste und letzte Instanz. So DemeliUs, Ratsurteile 12. Jeweils am Ende der beiden Phasen des Rechtsstreits beim Stadtrat, erfolgt aber ein Rechtszug an den Landesfürsten, nach 1507 wird gegen das Urteil des Wiener Stadtrats an das Kammergericht zu Wiener Neustadt "gedingt"; dessen Entscheidung aus 1509 ist ausschlaggebend für die Wiederaufnahme des Rechtsstreits am Wiener Stadtrat. Dazu LAMPL, Haupturkunden, Nr. 6112 (Anm. 3), der dort angeführte Nachfolgeband zu Bd. IV der Quellen zur Geschichte Stadt Wien ist nie erschienen. Der Wortlaut der Kammergerichtserklärung ergibt sich daher nur aus dem Original der Gerichtsurkunde (WStLA, HA-Urk. Nr. 1/1516, in: [http://vdu.unikoeln.de:8181/ mom/ATWStLA/HAUrk/6112/charter] (abgerufen 9. 8. 2011). Gegen das vom Stadtrat Mitte September 1516 erflossene Urteil wird abermals der Rechtsweg zum Landesfürsten, nämlich zum inzwischen 1510 für die niederösterreichischen Länder in Wien anstelle des Kammergerichts getretenen Regiment (Statthalterei und Hofrat), beschritten. Dessen Entscheidung ergeht Anfang Jänner 1524, kundgemacht vom Landesfürsten Anfang März und anschließend vom Stadtrat den Parteien bekanntgemacht: LAMPL, Haupturkunden, Nr. 6297a, Nr. 6298a und Nr. 6299.

${ }^{35}$ DemeLIUS, Ratsurteile 12. 
schlossen ist, sondern der landesfürstliche Stadtrichter Sigmund Pernfuß. ${ }^{36}$

\section{Gang des Verfahrens}

\section{A. Klagsbegehren und Klagsbeantwortung ${ }^{37}$}

\section{Klagsbegehren}

Das Begehren der Kläger gegen die Beklagte läuft auf die Rückerstattung der von Vorchtenauer als Willensvollstrecker („Geschaeftsherr" und "Vollfürer") des Geschäfts von Lukas Schnitzer übernommenen Betrags von 1.063 Gulden zu Handen von Paul Keck (als Vormund der minderjährigen Tochter des Lukas Schnitzer) ${ }^{38}$ und seiner Tochter Barbara (als Witwe nach Schnitzer) hinaus.

Der Anspruch stützt sich auch auf die eingangs bereits erwähnte Anordnung in Vorchtenauers Geschäft ${ }^{39}$, wonach diese Schuld von seiner Witwe Margarethe zu begleichen ist, und wofür er (Vorchtenauer) ihr (,seiner Hausfrau“) laut letztwilliger Verfügung auch ausreichend Vermögen hinterlassen habe (,wol sovil, das er ir gelassen, damit sü die Schulden bezalen möchte"). Besonders viel Gewicht legen die Kläger auch auf den Umstand, dass sich Margarethe den Nachlass von Vorchtenauer (,alles seines Guets, Erb- und varends [Gut]") eigenmächtigt angemaßt (,understanden“) habe. Sie sei unrechtmäßig vorgegangen, habe keine Zeugen beigezogen und auch die Obrigkeit davon nicht in Kenntnis

\footnotetext{
36 PERGER, Ratsbürger 169: Sigmund Pernfuß († um 1510/11), Stadtkämmerer (1497/99), Ratsherr (1509) und Stadtrichter (1503-1508).

${ }^{37}$ LAMPL, Haupturkunden, Nr. 5852.

38 Paul Keck ist dazu nicht nur als Vormund der minderjährigen Tochter des Lukas Schnitzer, sondern auch als der von diesem eingesetzte Willensvollstrecker legitimiert.

${ }^{39}$ LAMPL, Haupturkunden, Nr.5836a, vom 23. 8., publiziert am 8. 10. 1506.
}

gesetzt („,on Beiwesen Erberleut, on Willen und Wissen irer Öbrigkait"); sie habe auch kein Vermögensverzeichnis aufgenommen (,on alles Aufschreiben") und habe sich mehr als ein halbes Jahr die Nutzung des Nachlasses ihres früheren

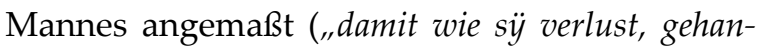
delt").

\section{Klagsbeantwortung}

Die Beklagte wendet gegen diese Vorwürfe ein, dass sie das Geschäft ihres früheren Mannes gar nicht annehmen wollte, sondern das Verlassenschaftsvermögen als privilegierte Pfandgläubigerin ${ }^{40}$ besitze (,,als ain gefreite Satzgelterin“), um die Abgeltung von ehegüterrechtlichen Ansprüchen („Heimstewer, Morgengab und Widerlegung") ${ }^{41}$, die sie mit ihrem früheren Ehemann vereinbart habe, sicherzustellen („damit sölhes vergnügt möcht werden"), wofür aber auch das zurückgebliebene Verlassenschaftsvermögen nicht zureichen würde.

\section{B. Replik der Kläger und Rechtfertigung der Beklagten ${ }^{42}$}

\section{Positionen der Kläger}

Die Kläger replizieren mit der Behauptung, dass ihnen von einer Heimsteuer oder Widerlegung zwar nichts bekannt sei, dass die Beklagte aber, selbst wenn sie ihrem früheren Mann solches Vermögen zugebracht hätte, wegen der halbjährigen rechtswidrigen Praxis diese Ansprüche verwirkt, "sich sölher Behelf unwirdig gemacht" habe. Auch sei es unglaubwürdig, dass Vorchtenauer nicht genügend Vermögen hinterlassen habe, damit ihre Ansprüche vergütet

\footnotetext{
${ }^{40}$ Dazu BRAUNEDER, Stillschweigende Hypotheken; vgl. DusIL, Grundpfandrechte 590.

${ }^{41}$ Dazu BRAUENDER, Ehegüterrecht 39ff., 51ff., 54ff. (Heiratsgut, Heimsteuer), 36f., 52ff., 54ff., 75 (Morgengabe), 52f., 56f. (Widerlegung), 75ff., 86ff. (Heiratsgabensystem); vgl.Brauneder, Eheliches Güterrecht.

${ }^{42}$ LAMPL, Haupturkunden, Nr. 5852.
} 
werden könnten. Wenn ihr daran gelegen wäre, den Wert des Nachlasses festzustellen, hätte sie ein Vermögensverzeichnis aufnehmen sollen. Nachdem dies aber unterlassen worden sei, habe sie sich ins Unrecht gesetzt und müsse nun mit Sanktionen rechnen (, die Recht thaeten ander schmechliche Vermutung wider sÿ“). Die Kläger regen bei den Beisitzern des Stadtrats an, nachdem das Klagebegehren den Anspruch einer minderjährigen Person (,Sachen der Unvogtperkait") betrifft, auch diesen gemäß (römisch-) gemeinem Recht als privilegiert (,von den gemainem Rechten gefreit") zu behandeln. Sollte der Rat aber erkennen, dass die Behauptung der Beklagten im geltenden Recht keine Grundlage habe („ob kain Gesetz oder Burger Recht waere verhanden"), so müsse der Rat nach Naturrecht entscheiden (,aus natürlichen Rechten und Vernunft erkhennen"). Da sich die Beklagte mehr als ein halbes Jahr eigenmächtig in den Besitz der Verlassenschaft ihres früheren Mannes gesetzt habe, würde ein solches Verhalten dem Zweck des Rechts zuwiderlaufen und es sei dem Täter daher jedes Rechtsmittel zu versagen (,,die Recht waeren sölhen Thaetern so hässig und widerwertig, das s̈̈ von inen hebeten alle sölh Behelf"). Sollte ein solches Verhalten für unwürdig und unredlich (,schmach und unlewnt") zu erachten sein, so würden die rechtkundigen (,rechtweisen“) Ratsbesitzer dies entsprechend $\mathrm{zu}$ ahnden wissen. Überdies habe sich die Beklagte einer Truhe mit Urkunden, auf Lukas Schnitzer lautend, bemächtigt (,,ainer Lad mit Briefen, auch Satz- und Geltbrief und ander brieflicher Urkunt auf [...] Lucasen Schnitzer [...] lautend [...] understanden").

Die Kläger beantragen daher, der Stadtrat möge "mit Recht und Urtail" erkennen, dass die Beklagte schuldig sei, zu Handen von Paul Keck als Vormund der Maria Schnitzer, und ihrer Mutter Barbara Haertl 1.063 Gulden, welche Vorchtenauer von Lukas Schnitzer erhalten habe, zu bezahlen, und die erwähnte Urkundentruhe dem Gericht auszuhändigen („überzeantwürten“).
Außerdem sei die Beklagte zum Ersatz (,Widerlegung") ${ }^{43}$ der Gerichtskosten zu verurteilen.

\section{Positionen der Beklagten}

In seiner Widerrede betont Dr. Wepp, seine Frau habe nach dem Tod von Vorchtenauer die Erbschaft ausgeschlagen, sei daher auch nicht als Erbin anzusehen und folglich nicht schuldig, sich für Vormundschaften ihres früheren Mannes zu verantworten. Bürgermeister Keck habe sich die Güter des Kindes von Lukas Schnitzer angeeignet (,understanden“), also alles, was dem Kind gehöre, bereits „emphangen“. Das Geschäft ihres früheren Mannes ginge sie nichts an, denn sie hatte ja davon bis vor kurzem gar kein Wissen. Offenkundig sei aber, betont Dr. Wepp, dass Vorchtenauer die Güter seiner Frau, welche mehr als 4.000 Gulden wert gewesen seien, während der Ehe "genossen, [... und laufend] verkauft" habe. Die Beklagte aber habe diese Güter zu Recht besessen, und zwar aus folgenden Titeln: die Liegenschaften als Miteigentümerin zu "gesambter Hand und Satzrecht"44; die Fahrnisse (,varenden“ Güter) als privilegierte Pfandgläubigerin ("gefreite Satzgelterin“); und das übrige Vermögen (,etlichs") als Alleineigentümerin (, als ir aigen Guet“). Ein Verzeichnis ihres Besitzes aufzunehmen sei sie als Eigentümerin bzw. Pfandgläubigerin jedoch nicht verpflichtet gewesen. Sie habe sich durch die Aneignung nämlich keinen (neuen) Besitz angemaßt (,understanden“), sondern sei "in der alten [posses] beliben", von Eigentümern werde rechtlich jedoch nicht gefordert, ein Verzeichnis ihres Vermögens aufzunehmen (,,wer kainer seines Guet schuldig Inventari zu machen"). Sie habe sich also „dadurch ihres Heiratsguets, Widerlegung, Morgen-

\footnotetext{
${ }^{43}$ Zur funktionell differenzierten Bedeutung des Rechtswortes „Widerlegung“: BRAUNEDER, Widerlegung.

${ }^{44}$ Zum mittelalterlichen Miteigentum im Rechtsleben von Wien: BRAUNEDER, JARITZ, Stadtbücher 1, 19-21; zur Satzung: BRAUNEDER, Ehegüterrecht $77 \mathrm{f}$.
} 
gab und andrer zuebrachter Gueter nicht entsetzt" und habe auch "nicht wider Eer gehandelt oder schmechlich Vermuttung auf [... sich] geladen".

Der Bürgermeister dagegen wäre verpflichtet gewesen, ein Inventar aufzunehmen, denn er habe sich das Vermögen seines Mündels angeeignet (,sich [...] des Kinds Güeter [...] understanden") und "Trühen und anders" aus der Stadtschreiberkanzlei im Rathaus abtransportieren (,austragen“) lassen.45 Sie, die Beklagte, habe keinerlei Kenntnis gehabt, was davon erfasst worden sei (,waer ir nicht wissen, was darinn waer gewesen"). Sie selbst habe "sich nichts understanden" ohne Kenntnis („Wissen, Willen und Haissen") des Bürgermeisters und habe stets nach seinen Anleitungen gehandelt (, seinem Rat gehandlt"). Ihre Forderung gegen den Nachlass ihres früheren Mannes belaufe sich auf mehr als 4.000 Gulden Wert, welche sie aus Anlass der Eheschließung und während der Ehe eingebracht habe.

Über den Vorwurf der Kläger, sie halte unrechtmäßigerweise eine Lade mit Urkunden ihres früheren Mannes zurück, zeigt sich die Beklagte verwundert (wegen der "Lad mit Briefen halben befrömbt s̈̈ sich"), weil sich Dr. Wepp dem Bürgermeister gegenüber schon bereit erklärt habe, diese jederzeit zu übergeben (, ,alzeit sich [...] erboten hiete [diese] fürzebringen"). Abschließend behält sich Margarethe Rietenberger noch vor, falls der Nachlass nicht zureichen sollte, um ihre Forderung von gut 4.000 Gulden zu befriedigen, auch Ansprüche auf die Liegenschaften ihres früheren Mannes ${ }^{46}($,Zuesprüch, $[\ldots] z u[\ldots]$ Steffans Heuser und Güeter"), insbesondere auf das gemeinsame Haus am Kienmarkt ${ }^{47}$, geltend zu machen (,zu seiner Zeit für[zu]bringen“).

\footnotetext{
${ }^{45}$ LAMPL, Haupturkunden, Nr. 5844, Notariatsinstrument vom 15./16. 1. 1507.

${ }^{46}$ Dazu unten IV.B. unter der entsprechenden Rubrik des Verlassenschaftsinventars.

${ }^{47}$ Haus "zur großen Landskrone“ (alte Konskriptionsnummer 548, Heute Landskrongasse Nr.1-3,
}

\section{Duplik der Kläger und Einwendungen der Beklagten}

\section{Positionen der Kläger}

Nach dem Vorbringen von Dr. Wepp liegt es nun wieder an den Klägern, die Ausführungen der Beklagten zu entkräften und die eigenen Positionen zu vertreten. 48

Bürgermeister Keck ${ }^{49}$ greift zunächst die Behauptung der Beklagten auf, dass es ihr nicht als Annahme der Erbschaft zugerechnet werden könne, wenn sie sich den Nachlass ihres früheren Mannes ohne Inventarisierung angeeignet (,seines gelassen Guets on ain Inventarj underwunden") habe. Dies gehe auch klar aus dem geltenden Recht hervor (wie "die Recht klaerlich anzaigeten"). Und selbst wenn sie die Erbschaft nicht angenommen haben sollte, könnte dies die Haftung für die Vormundschaftsschulden des Erblassers nicht ausschließen (,,entgült sü nicht der Gerhabschaft [...] Steffans").

Anschließend hebt Keck hervor, dass das Geschäft von Vorchtenauer (mit den darin enthaltenen Anordnungen) rechtmäßig bestehe und "nach Gewonhait und Statrechten" gültig sei. Durch die eigenmächtige Inbesitznahme des Nachlasses habe sich die Beklagte aber auch für die darin enthaltenen Schulden haftbar gemacht („sich selbs verbunden [...], die Gelter zu bezalen“). Sie habe in Bezug auf die 1.063 Gulden, welche sie von ihrem früheren Mann übernommen hat, und welche den Klägern, Frau Haertl und ihrer minderjährigen Tochter („irem ungevogten Kind"), zustehen, den Besitz - gleichsam durch Besitzkonstitution - fortgesetzt (,Posseß [...]

Bauernmarkt Nr. 13, Wilpretmarkt Nr. 10), von Vorchtenauer 1504 vom Konvent des Klosters St. Hieronymus erworben: HARRER-LUCIENFELD, Wien 420, 746.

${ }^{48}$ Das Verfahren zeigt insofern zwar schon Einflüsse des römisch-gemeinrechtlichen Artikelverfahrens, es wird aber ausschließlich mündlich verhandelt. Vgl. WESENER, Einflüsse 58; VOLTELINI, Rezeption 91.

${ }^{49}$ LAMPL, Haupturkunden, Nr. 5852. 
continuirt"), sodass sie nun auch verpflichtet sei, diesen Betrag auszufolgen (,schuldig waer zu bezalen").

Gegen die von der Beklagten angegeben Rechtstitel für den rechtmäßigen Besitz des Nachlasses wendet Paul Keck vor allem ein, dass das von ihr behauptete Pfandrecht nicht anerkannt und „ir kains satz" zugestanden werde, weil sie nach Gewohnheitsrecht nicht als Pfandgläubigerin

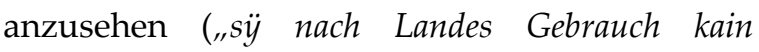
Satzgelterin") sei. Und wenn das (römisch)gemeine Recht (,kaiserliche Recht") der Ehegattin am Vermögen des Mannes wegen ehegüterrechtlicher Ansprüche ${ }_{,}, u m b$ Heiratgut und Haimstewer") auch Sicherungsrechte zuerkenne („der Frawen des Manns Güeter thaeten verphlichten"), so gelte dies in gleicher Weise auch für Ansprüche von Minderjährigen (,gleichwol thaeten verphlichten die kaiserlichen Recht den ungevogten Kindern"). Es seien also in diesem Fall beide Seiten gleichermaßen privilegiert (,baide in sölhen geleich gefreit"). Und selbst wenn dem Mündel nach (römisch-)gemeinem Recht gegen die Beklagte kein solches Privileg (,ob das Kind von gemainen Rechten kain [...] sölher Freihait") zukommen sollte, so hätte sich die Beklagte doch dieses Vermögen nicht aneignen dürfen (,hiet sü [..] nicht mügen [.. zu] understeen"). Die Kläger wissen aber gar nicht, wie hoch dieses Vermögen sei (,wieviel Werts sÿ sich understanden hiet"), und ob die eigenmächtige Aneignung für die Beklagte auch strafrechtliche Sanktionen nach sich ziehe (,ob ir Missetat daraus erwüchs"). Jedenfalls werde den Angaben der Beklagten über den Wert des von ihr in die Ehe eingebrachten und danach vermehrten Vermögens mit mehr 4.000 Gulden kein Glauben geschenkt.

\section{Positionen der Beklagten}

Die Beklagte hält den klägerischen Einwendungen Folgendes entgegen:50 Sie habe von dem Geschäft ihres früheren Mannes erst am 6. März 1507 - aus Anlass der Klage - erfahren. Es könne ihr daher nicht der Vorwurf gemacht werden, dass sie das Nachlassvermögen als Erbin an sich genommen (, sich der Güeter als ain Erb hiet understanden") habe, wo sie doch überhaupt nicht wissen konnte, dass sie in dem Geschäft von ihrem früheren Ehemann als Erbin berufen worden sei. Sie habe dieses Vermögen eben aus anderen Gründen in Besitz genommen, nämlich aufgrund der bereits angeführten Titel. ${ }^{51}$ Daher sei sie auch nicht verpflichtet, ein Vermögensverzeichnis aufzunehmen, und zwar aus folgenden Gründen („Ursachen“): Sie sei nicht Erbin dieses Vermögens, sondern privilegierte Gläubigerin ("gefreit Gelterin"), und habe den Nachlass ihres früheren Mannes daher "titulo oneroso" inne - wegen der Forderungen gegen ihren früheren Mann. Inzwischen habe sie sich explizit der Berufung zur Erbin entschlagen und sei auch deswegen nicht verpflichtet, ein Vermögensverzeichnis aufzunehmen. Anders liege der Fall etwa bei einem Willensvollstrecker ("Geschaefftherr oder testamentari“): Er sei nach geltendem Recht („nach Ordnung des Rechten“) zur Inventarisierung verpflichtet, insbesondere dann, wenn er mit Nachlassvermögen disponiert, "das Gut einämb, das besichtiget, auf- und zuesperret", so wie eben Paul Keck.

Sodann bringt die Beklagte noch einen Eventualeinwand vor: Selbst wenn sie wirksam Erbin geworden sein sollte, und daher auch verpflichtet gewesen wäre, ein Vermögensverzeichnis aufzunehmen, könnte sie dies immer noch rechtzeitig bewirken, denn das geltende Recht sehe dafür eine Frist von drei Monaten nach Eröffnung des Geschäfts vor; und diese Frist sei

\footnotetext{
${ }^{50}$ LAMPL, Haupturkunden, Nr. 5852.

${ }^{51}$ Dazu oben II.B.2.
} 
- nachdem die Eröffnung des Geschäfts erst am 6. März erfolgt ist - noch offen. In diesem $\mathrm{Zu}$ sammenhang weist die Beklagte auch darauf hin, dass es ihr faktisch unmöglich gewesen sei, ein Inventar aufzunehmen, weil die Verlassenschaft ihres früheren Mannes anfänglich in Sperre genommen und mit dem Petschaft des Bürgermeisters versiegelt gewesen sei; später habe der Kläger aber einen Teil dieses Vermögens separiert und ihr den Rest überlassen.

Gegen den Bürgermeister richtet die Beklagte sodann den Vorwurf, er habe Vorchtenauers Testament zunächst unterdrückt und das Geschäft ihres früheren Mannes „erst lang nach seinem Tod", und nachdem sie sich sein Nachlassvermögen bereits angeeignet hatte (,,sï sich der übrigen Güeter understanden hiet"), offengelegt („herfürbracht“). Es wäre aber unrechtmäßig, wenn ein Willensvollstrecker das ihm anvertraute Geschäft "haimlich öffne [im] Voraus" - ohne Erben und Obrigkeit einzubinden (,,hinter Ruckh des Erben oder [...] der Öbrigkait"). Der Bürgermeister habe ihr das Geschäft ihres früheren Mannes erst vor ein paar Tagen in Abschrift übergeben (,dasselb Geschaefft [...] etlich Täg vor [...] zugeschickt").

Unter Hinweis auf den Verkauf ihres in die Ehe eingebrachten Vermögens (Fahrnis, Silber, und Silbergeschirr sowie anderen Hausrats, "Eisen, Salzkaufmanschaft, Wein und Getraid"), alles in allem mehr als 4.000 Gulden wert, versucht sie ihr Vorrecht am Nachlass ihres früheren Mannes als dessen hauptsächliche, und vor allen anderen privilegierte Gläubigerin („mit sölhen iren Schulden für all ander Gelaubiger gefreit") zu rechtfertigen. Sie erklärt dazu, dass ihr Heiratsgut gemäß Heiratsabrede ursprünglich zwar nur 900 Gulden "hungrisch“52 betragen habe, dass es aber danach dadurch vermehrt worden sei, dass sie ihrem früheren Ehemann auch ihr übriges Vermögen überlassen habe (, laut Abred der Hei-

\footnotetext{
${ }^{52}$ Ungarische Golddukaten.
}

rat, [...] waer doch nachmalen das Heiratgüt gemert worden und hiet [sie] im all ir Guet vergünt").

Der Behauptung der Kläger, dass das Mündel am Vermögen seines Vormunds wegen seiner Forderungen ebenso privilegiert sei wie die Ehefrau wegen ihrer Ansprüche auf das in die Ehe eingebrachte Vermögen gegen ihren Ehemann („,ie Gerhabkinder waeren gegen iren Gerhaben geleich gefreit, wie die Fraw umb ir zugebracht Guet"), hält die Beklagte entgegen, dass sie mit ihrer Forderung dem Mündel und anderen Gläubigern vorangehe (,ain Fraw waer mit irem zugebrachten Güt für alle Kinder und ander Gelter gefrëyt"), weil ihr Pfandrecht Priorität genieße (,waere doch ir Phandschaft [...] elter"); die Mutter des Mündels, Barbara Haertl, sei mit ihrer Forderung aber überhaupt nicht privilegiert ("nicht gefreit").

Sodann verwahrt sich die Beklagte gegen die Unterstellung der Kläger, sie habe sich des Vermögens ihres früheren Mannes heimlich und räuberisch (,,als ain pr[a]edo") bemächtigt, und zwar unter abermaligem Hinweis auf die bereits dargelegten Titel: An einem Teil dieses Vermögens sei sie als Miteigentümerin („,kraft gesambter Hand"), an einem anderen als Alleineigentümerin (, ,aigen Guet") berechtigt; der Rest stelle das von ihr in die Ehe eingebrachte väterliche und mütterliche Erbe dar. Sie habe dieses Vermögen mit Einverständnis (,,aus Wissen, Willen und Haissen“) des Bürgermeisters als der zuständigen Obrigkeit und als Willensvollstrecker („Geschaefftiger") von Vorchtenauer übernommen, wenngleich Keck dies nun abstreiten wolle. Bald nach ihrer Genesung sei sie aus dem Rathaus in ihr eigenes Haus umgezogen und habe, was an Vermögen ihres früheren Ehemannes noch vorhanden war, mit sich genommen; der Bürgermeister habe ihr dazu zwar nicht ausdrücklich geraten, diesem Vorgehen aber auch nicht widersprochen (,,hiet er ir sölhs nit geraten [...], so hiet es im gezimbt, sölhs zu widersprechen"). Auch könne man nicht sagen, sie habe gegen seinen Willen gehandelt, wo er doch 
selbst mit seinen Amtsleuten einen Teil dieses Vermögens unmittelbar davor wegführen habe lassen.

Der Behauptung der Kläger, wonach sie den Besitz des Vermögens ihres früheren Mannes und daher auch in Bezug auf die 1.063 Gulden, welche die Kläger beanspruchten ("die der Widerparthei zugehörten“) - bloß fortgesetzt und nicht neu begründet ("die posseß der Güter continuirt [...] und kain newe posses angefangen"), hätte, hält sie entgegen, dass vom Bürgermeister zugegeben worden sei, er habe während der Zeit ihrer Erkrankung das Vermögen des minderjährigen Kindes wegführen lassen (,hiet ÿetzo [...] bekennt [...] daz er, dieweil sÿ kranckh waer gewesen, des Kindes Güeter hiet ausgefürt"). Da besagte 1.063 Gulden dem Kind zuzurechnen seien, habe sie den Besitz ihres Mannes an dieser Summe nicht fortgesetzt, sondern der Bürgermeister habe ihren Besitz durch sein Handeln unterbrochen (", die Possess derselben Summa [...] interrumpirt"). Das vom Bürgermeister behauptete Besitzkonstitut beziehe sich daher nur auf ihr eigenes Vermögen („ire Güeter, daran sü an Nutz [und] Gewer stuend"), das daher auch nicht als fremdes Vermögen angesehen werden könne.

Den Vorwurf der Kläger, sie wüsste nicht einmal, wie hoch das Vermögen sei, das sie von ihrem früheren Mann an sich gezogen hatte (,s s̈y wüßte nicht was und wievil Güeter sü sich understanden hiet"), wehrt sie mit dem Einwand ab, es sei so viel als vom Bürgermeister und seinen Amtsleuten im Rathaus zurückgelassen worden sei. Sie habe davor keine Gelegenheit gehabt, auf dieses Vermögen zuzugreifen, weil die Räume versiegelt (,verpetschad“) gewesen seien.

\section{Triplik der Kläger und abschließende Rechtfertigung der Beklagten}

\section{Positionen der Kläger}

Für die Kläger muss nun Paul Keck den Behauptungen der Beklagten entgegnen. ${ }^{53}$ Er bringt zunächst vor, es sei unerheblich, ob sie als Erbin anzusehen wäre oder nicht. Weil sie sich eigenmächtig („on Mitl der Rechten“), ohne Wissen der Obrigkeit und ohne schriftliche Aufzeichnungen darüber gemacht oder Zeugen beigezogen $\mathrm{zu}$ haben ("Beiwesen Erberleut") in den Besitz des Nachlassvermögens ihres früheren Ehemannes gesetzt habe, sei sie billigerweise verpflichtet, dessen Schulden an Ehefrau und Kind des Lukas Schnitzer zu bezahlen.

Die Behauptung der Beklagten, sie sei zur Inventarisierung rechtlich nicht verpflichtet gewesen, so wie etwa ein Willensvollstrecker oder ein Vormund, wäre ein "s[ch]wach[es ...] Argument". Den von der Beklagten dafür vorgebrachten Gründen, hält der Kläger folgende Positionen entgegen: Sie habe erstens aufgrund ihres eigenmächtigen Vorgehens jeden Rechtsanspruch („all ir Gerechtigkait") verloren und sei daher verpflichtet, diese Schulden zu erfüllen (,die Gelter schuldig zu bezalen“). Außerdem habe sie sich dadurch der Sanktion ("Peen und Straff") der kaiserlichen Konstitution (,kaiserlicher Gesetz"), welche mit den Worten "Si quis in tantam" beginnt ${ }^{54}$, ausgesetzt: Danach verliere jeder, der sich ein Pfandrecht ("Satz oder Phant") ohne Zustimmung von Obrigkeit oder Pfandeigentümer ("der des Phands Aigenthumb hiet") anmaßt, nicht nur das Pfandrecht, sondern verfalle auch

\footnotetext{
${ }^{53}$ LAMPL, Haupturkunden, Nr. 5852.

${ }^{54}$ C 8,4,7. (unde vi). Konstitution aus 389: Jede Besitzentziehung ist verboten, selbst wenn sie im Wege der Selbsthilfe gegen eine verbotene Besitzentziehung erfolgt; der Täter verliert zur Strafe sogar sein Eigentum oder muss einen Betrag in Höhe des Sachwertes leisten.
} 
den Folgen dieses Gesetzes. Dasselbe gelte nach allgemeinem Stadtrecht („gemainer Stat Rechten und Brauch"), wonach niemand ohne Zustimmung des Grundherrn und ohne Beweisführung im Rat ein Pfandrecht behaupten dürfe (,,möchte sich kainer berümen aines Satz, der mit Grundherren Handen nicht gefertigt waer"). Die Beklagte könne daher nach geltendem Recht kein Pfandrecht behaupten und sei daher auch nicht als Pfandgläubigerin („kain Satzgelterin“) zu bezeichnen. Zweitens sei es unerheblich, ob die Beklagte von ihrer Berufung zur Erbin Kenntnis gehabt habe oder nicht - dies diene nicht zur Sache; allein die Aneignung des Nachlasses würde sie verpflichten, die Gläubiger zu befriedigen (,den Geltern Bezalung zu thun"). So hätten auch die Legisten entschieden („,spraechen die Lerer kaiserlicher Rech$t^{t e n}$ "). Der dritte Grund, den die Beklagte vorgebracht habe, berühre ihn, Paul Keck, allein, nämlich dass er die Sperre über das Nachlassvermögen von Vorchtenauer während der Erkrankung der Beklagten ohne ihr Wissen aufgehoben habe. Dem stehe aber entgegen, dass er nur aus Fürsorge für seine minderjährige Enkelin und deren Mutter gehandelt habe. Allein der Umstand, dass diesen das Vermögen, das Vorchtenauer zu seinen Handen genommen habe, nicht verloren gehe, sei der Beweggrund gewesen, das, was seiner Tochter oder ihrem Kind gehört, in gerichtlich versperren und wegbringen zu lassen. Viertens habe die Beklagte dadurch, dass sie sich den Nachlass ihres früheren Mannes ohne Inventarisierung angeeignet habe, unrechtmäßig gehandelt; wie verwerflich dieses Handeln gewesen sei (,wie schimphlich das waer"), werde das Gericht zu ermessen haben. Den fünften Grund hätte die Beklagte, hebt der Kläger hervor, gar nicht vorgebracht, wenn nicht er aus Vorchtenauers Vermögen die seiner Tochter und Enkelin zugehörigen Sachen gerichtlich in Sperre genommen und versiegeln (,versperren [...] und [...] mit Gerichtshanden verpetschaften") hätte lassen.
Sodann geht Bürgermeister Keck auf die Behauptung der Beklagten ein, dass es viel eher an ihr gelegen wäre, ein Inventar aufzustellen, weil sie sich nicht mehr Verlassenschaftsvermögen aneignen habe können, als er bei ihr (,in den Zimmern") zurückgelassen hätte. Der damit in Verbindung stehenden Behauptung der Beklagten, er habe das Geschäft seines Schwiegersohnes heimlich und hinter ihrem Rücken vor den Rat gebracht und öffnen lassen (",haimlich und hinder rügkh waere gewisen und geöffnet"), setzt Keck entgegen, dass er durchaus dem Stadtrecht und Herkommen sowie dem allgemeinen Gerichtsgebrauch gemäß (,wie stat Recht und Herkommen auch der Brauch gemains Rat wern") vorgegangen sei. Doch ungeachtet dessen, ob sie sich nun sofort nach Berufung zur Erbin der Erbschaft entschlagen, oder sich später unrechtmäßig (,on Mitl der Rechten“) das Nachlassvermögen angeeignet habe, ändere dies nichts daran, dass sie verpflichtet gewesen wäre, die nachgelassenen Schulden ihres früheren Mannes zu übernehmen (,waer s̈̈ dennoch die Gelter schuldig zu haben").

Der Bestand einer Forderung von 4.000 Gulden gegen den Nachlass, welche die Beklagte ihrem früheren Mann während der Ehe zugebracht haben soll, wird von Keck in Zweifel gezogen: Er glaube weder, dass das zugebrachte Vermögen diesen Wert gehabt habe, noch dass Vorchtenauer etwas davon - wie die Beklagte behauptet - auf eigene Rechnung verkauft habe; und wenn doch, dann stelle sich die Frage, an wen, und ob jemand noch etwas aus einem Kauf schulde. Selbst wenn die Beklagte ihm dieses Vermögen auch tatsächlich zugebracht haben sollte, dann wäre dies mit der Aneignung des Nachlasses abgegolten („mit seinem gelassen Gut bezalt") - sie bleibe daher verpflichtet, die inbegriffenen Schulden zu übernehmen (,die Gelter schuldig zu bezalen").

Der Erklärung der Beklagten, dass sie mit ihrer Forderung vor allen anderen Gläubigern privilegiert (,für all ander Gelter gefreit“) sei, hält Keck 
entgegen, dass sie wegen ihres eigenmächtigen Vorgehens dieses Vorrecht - und damit auch den Pfandbesitz - verwirkt („wann sü gefreit waer, hiet sü ir Freihait verloren mitsambt [... der] Phandschaft") habe. Dem Begehren der Beklagten, zugunsten ihrer Forderungen noch anderes, zufällig auftauchendes Vermögen von Vorchtenauer gerichtlich einziehen $\mathrm{zu}$ lassen (,begeret Erlaubnuss, ander Güeter [...] Steffans zu völliger Bezalung einzuziehen"), stellt Keck den Vorwurf entgegen, es sei gerichtsbekannt, dass die Beklagte zur Aneignung von Vorchtenauers Nachlassvermögen (,Underwindung seines [...] gelassen Guts") die Erlaubnis des Rats nicht eingeholt habe - doch nun, nach unrechtmäßigem Handeln, wolle sie eine solche Erlaubnis.

Zur Behauptung der Beklagten, wonach ihr Heiratsgut anfänglich zwar nur 900 Gulden "hungrisch"55 betragen habe, aber während der Ehe um ein Mehrfaches vermehrt worden sei, meinen die Kläger, dass dieser Betrag ursprünglich vielleicht vereinbart (,abgeredt") gewesen sein mag, doch glaube man nicht, dass dieser Betrag auch tatsächlich übergeben (in „par gelt [...] zubracht") worden sei: Vereinbarung und Übergabe ("Abred und Zubringen“) seien eben zwei verschiedene Dinge; und es frage sich auch, wie ein Vermögen sich vermehrt haben konnte, wenn es gar nicht übergeben worden ist. Auf die Einwendung der Beklagten, dass ihre Forderung älter sei als jene von Kecks Enkelin, erwidert dieser, es komme in diesem Fall nicht auf einen zeitlichen Vorrang an, sondern auf den Titel der Privilegierung (,wann in disem Val die Freihait der Gelter nicht von Elter wegen der Schuld, [...] sondern aus den Ursachen darauf die Freiheit gründt waer"). Im vorliegenden Fall verhalte sich der Grad der Privilegierung des Kindes zu dem der Beklagten etwa wie 4:2, man würde für das Vorrecht des Kindes also vier Gründe finden, für jenes der Frau aber nicht

\footnotetext{
${ }^{55}$ Ungarische Golddukaten.
}

einmal zwei (,Ursach warum das Kind gefreit solt sein [...] vier, so wie nicht zwo [...] in Ermessen der Frawen Frë̈hait").

Schließlich weist Keck auch die Behauptung der Beklagten zurück, er habe ihr zu diesem Handeln geraten; und bringt eine ironischen Vergleich: Wann immer er ihr etwas Gutes geraten habe, hätte sie es nicht befolgt (,wann er ir vil Guts hiet geraten, nimmer gevolgig gewesen"). Denn, wenn man Frauen die Freiheit der Entscheidung lasse, dann würden sie lieber in ein

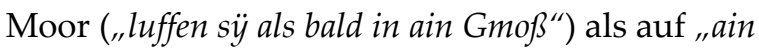
grüne Wissen" laufen.

\section{Positionen der Beklagten}

In seiner Widerrede für die Beklagte setzt nun deren Ehemann Dr. Wepp ${ }^{56}$ den einzelnen Positionen der Klägerseite ihre Einwendungen entgegen: Margarethe habe nach dem Tod ihres Vaters Vorchtenauer geheiratet; dieser habe sich das von ihr eingebrachte Vermögen, bestehend aus Silber, Schmuck ("Klainat"), Urkunden („Brief"), Siegel, Forderungen („Schulden“), Eisen, Salzprodukte („Salzkaufmanschaft"), Wein und Getreide, ohne ein Inventar aufzunehmen, angeeignet (,understanden") und es wieder verkauft. Bei seinem Tod habe er bloß noch "etlich Klainat und Silbergeschirr" von dem eingebrachten Vermögen seiner Frau ${ }^{57}$ besessen. Den Erlös für das übrige von ihm veräußerte Vermögen habe Vorchtenauer für den Erwerb von Liegenschaften in Wien (,Erb, die er hie kauft hiet") verwendet. Bei seinem Tod sei Margarethe lebensgefährlich erkrankt und der Bürgermeister habe die Zimmer von Vorchtenauer im Rathaus versiegeln lassen. Später sei aber auf seine Veranlassung - was Keck ja auch zugebe - Vermögen von Schnitzers Kind "ausgeführt" worden, und dennoch fordere er noch weitere 1.063 Gulden,

\footnotetext{
${ }^{56}$ LAMPL, Haupturkunden, Nr. 5852.

${ }^{57} \mathrm{Vgl}$. dazu unten einzelne dafür typische Gegenstände im Nachlassinventar von Vorchtenauer unter der Rubrik Kleidung und Schmuck.
} 
welche ebenfalls dem Kind und dessen Mutter zukommen sollen. Nach einiger Zeit habe der Bürgermeister die Beklagte aufgefordert, die Wohnung Rathaus zu räumen. ${ }^{58}$ Auf seine Anordnung sei die Sperre über das Nachlassvermögen von Vorchtenauer aufgehoben (,die Sigl und Pedschad von den Türn gethan") worden und die Beklagte habe ihr Vermögen wegbringen (,hiet sü haissen ir gut ausfüren“) lassen; Keck habe ihr sogar den eigenen Wagen zur Verfügung gestellt.

Sodann hebt Dr. Wepp hervor, dass Vorchtenauer sein Geschäft eigenhändig verfasst und versiegelt (seinem Stiefvater) Bürgermeister Keck übergeben habe. Dieser habe es geöffnet (,aufgebrochen“) und im Rat vorgelegt. Die Witwe sei davon aber nicht in Kenntnis gesetzt (,,ir nie darzu verkünt") worden. Erst nach ihrer Wiederverehelichung sei es ihr vorgelegt worden. Der Inhalt des Geschäftes ihres früheren Mannes habe ihr also nicht bekannt gewesen sein können, sie habe sich der darin enthaltenen Berufung zur Erbin daher auch erst entschlagen, nachdem sie Kenntnis davon erlangt habe.

\footnotetext{
${ }^{58}$ Nachdem der Vorwurf aufgebracht worden war, dass Ratsdiener und Schrannenschreiber "für allfällige Abgänge verantwortlich" zu machen wären, ließen die beiden städtischen Bediensteten am 15./16. 1. 1507 vor öffentlichem Notar unter Beiziehung der damals anwesenden Zeugen ein Protokoll über diese Vorgänge, deren Datum nicht mehr feststellbar ist, aufnehmen: LAMPL, Haupturkunden, Nr. 5844. Der im Rathaus - am Arbeitsplatz - verbliebene Nachlass des Stadtschreibers wurde - wohl bald nach dem Ableben von Vorchtenauer - auf Anordnung von Bürgermeister Paul Keck und Stadtrichter Sigmund Pernfuß durch vom Ratsdiener im Beisein des Schrannenschreibers genommen in "Sperre“. Später hat der Bürgermeisters einen Teil davon, nämlich „des Lucasen Schnytzers saligen verlassen Hab und Gütt" (zwei große Truhen und zwei Laden), das Vorchtenauer "in Behaltnüs gehabt", vom Rathaus ins Bürgermeisterhaus verbracht, "damit es unverkhumert [...] desselben Lucasen [...] unvogtbaren Khindern beleibe".
}

Den Einwendungen der Kläger gegen die von der Beklagten vorgebrachten Gründe für ihren rechtmäßigen Besitz an Vorchtenauers Nachlass stellt Dr. Wepp nun folgende Behauptungen gegenüber: Erstens sei die Argumentation der Beklagten gegen die Anwendung des Gesetzes "Si quis in tantam" unwiderlegt geblieben und daher als richtig anzunehmen. Sie habe nämlich mit Wissen der Obrigkeit dieses Vermögen in Besitz genommen, der Bürgermeister selbst habe die Sperre des Nachlasses, ohne von ihr dazu aufgefordert worden zu sein (,on ir Begeren“), aufgehoben (,aufgespert"); er habe sie aufgefordert, die Sachen - samt Wein in Fässern (, die wein geprest, die vässer gebunden“) - wegzubringen, und zwar unter Mitwirkung des städtischen Unterkämmerers (,,mit dem Undercamrer “). Nach Vorchtenauers Tod sei aber Vermögen zurückgeblieben, an dem auch die Beklagte berechtigt gewesen sei, denn dieses Vermögen habe Vorchtenauer von jenem Geld erworben, das von ihr in die Ehe eingebracht worden sei. Sie habe dieses Vermögen dann nach dem Tod ihres früheren Mannes zur Sicherung ihres Anspruchs auf das Heiratsgut in Besitz (,,aus [...] Freihait Heiratsguts") genommen, damit aber bloß jenes Vorrecht wahrgenommen, das jeder Frau nach dem Tod des Ehemannes („nach Abgang irs Manns") zukomme - und zwar, ohne deswegen der Strafe des Gesetzes "Si quis in tantem" zu verfallen. Dieses Gesetz komme auch nur dann zur Anwendung, wenn sich jemand einen Besitz unrechtmäßig angemaßt habe (, ,sich ainer newen posseß understanden"). Sie, die Beklagte, aber sei in dem gemeinsam mit ihrem früheren Ehemann begründeten Besitz (,neben irm vorigen $[. .$.$] hauswirt alzeit [. .$.$] in posseß")$ verblieben. Das Gesetz "Si quis in tantam" habe daher eher auf Bürgermeister Keck Anwendung $\mathrm{zu}$ finden, weil er einige Truhen und andere Sachen aus der Wohnung von Vorchtenauer im Rathaus wegbringen habe lassen. Dies sei auch in einer Notariatsurkunde (,in ain Instrument") aufgrund von Zeugenaussagen beurkundet 
worden. Der Bürgermeister habe zwar angegeben, dass er ausschließlich das Vermögen seines Enkelkindes wegbringen habe lassen, er habe sich dieses Vermögen aber unrechtmäßig angeeignet und sei daher selbst der erwähnten Strafe verfallen. Es könne auch nicht die Rede davon sein, dass er in seiner Funktion als Bürgermeister von der Anwendung dieser Strafe auszunehmen sei, denn er trete in diesem Fall nicht als Richter, sondern als Vormund und daher als Partei auf. Der Einwand von Keck, dass Vorchtenauers Vermögen als herrenloses Gut („vacantia“) zu betrachten sei, wird von Dr. Wepp mit dem Argument zurückgewiesen, dass der Bürgermeister vom Geschäft Vorchtenauers als dessen Willensvollstrecker Kenntnis gehabt habe. Die Strafe des Gesetzes "Si quis in tantam" trete aber auch dann ein, wenn sich jemand einen herrenlos gewordenen Besitz aneigne (,ainer ledigen Possession understünd"). Was immer der Bürgermeister also gegen seine Frau ins Feld führe (,wider sü erdenckhen [...] möchte"), werde nur ihm selbst Schaden zufügen (,thet mer wider in"). Denn nicht sie sei verpflichtet gewesen, ein Inventar aufzunehmen, sondern er, der Kläger als Willensvollstrecker (,als [...] Geschaeftiger") und als derjenige, welcher den Nachlass geöffnet und einen Teil davon abgesondert („eröffnet und davon gefürt") habe. Keck wäre als Willensvollstrecker ja auch verpflichtet gewesen, die Bezahlung von Vorchtenauers nachgelassenen Schulden zu bewirken (,waer schuldig ir völlige Bezalung zu thun"). Keck habe sich im Namen seiner Enkelin des im Besitz von Vorchtenauer und der Beklagten befindlichen Vermögens bemächtigt (,der Gueter [...] on ir Willen [...] understanden") und sie dadurch aus ihrem Besitz verdrängt (,s Possession entwert"). Keck wäre daher zu verpflichten, der Beklagten, diesen Besitz zurückzuerstatten (,waer er ir schuldig, [...] wider zu geben"). Die Bestimmung des Stadtrechts, wonach eine eigenmächtige Pfandnahme unwirksam („kain Satz on des Grundherren Handen kreftig") wäre, sei für diesen Fall nicht anwendbar, sondern nur bei vertraglich begründeten Pfandrechten („Sätze[n], die durch Verträg beschaehen"), jedenfalls aber nicht für den Fall einer Pfandnahme zur Sicherung des Heiratsguts der Ehefrau („,so umb ainer Frawen Heiratgut waeren, verphent"). Diese Forderung betreffe aber nicht bloß 900 Gulden "hungrisch" an Heiratsgut samt Widerlegung und Morgengabe, sondern auch die spätere Vermehrung dieses Vermögens, auf welche sich das Pfandprivileg nun erstrecke (,sölh Merung des Heiratguets wer gleich gefrait mit dem Heiratgüet").

Auf die Behauptungen der Kläger, Wissen oder Nichtwissen der Beklagten über ihre Berufung als Erbin trüge zur Sache nichts bei, und die Beklagte hätte lieber den Rat von gelehrten Juristen beiziehen sollen (,s̈̈ solt Rat haben gephlegen von [...] Gelerten"), erwidert Dr. Wepp, die Beklagte hätte wohl gerne den Rat gelehrter Leute in Anspruch genommen, der Bürgermeister habe aber niemanden über den Inhalt von Vorchtenauers Geschäft informiert (,hiet niemant gesagt, was in dem Geschaefft"). Die Beklagte sei sich also über den Grund ihrer Berufung zur Erbin - ob kraft Gewohnheit oder gemäß Geschäft („Wissen wer not, aus was Ursach [...] [sie] Erb wer, als ain gesipter Erb oder aus ainem Gescheft") - nicht bewusst gewesen. Sie habe sich daher durch die Aneignung des Nachlasses nicht unrechtmäßig in eine Erbschaft eingemischt.

Anschließend geht Dr. Wepp auf den Umstand ein, dass Keck einige Kisten aus dem Rathaus wegschaffen (,ausfüren [... und] aus dem Rathaus tragen") habe lassen - ohne darüber eine Aufzeichnung („kain Aufschreibung“) zu veranlassen. Für Dr. Wepp ist nun fraglich, warum der Bürgermeister, wenn er schon nicht aufzeichnen habe lassen, was zugunsten des Kindes an Vermögen abgesondert worden ist, dann nicht als Vorchtenauers Willensvollstrecker ("Geschaefftiger") das Vermögen, das im Nachlass von Vorchtenauer verblieben ist, inventarisieren 
habe lassen. Wäre ein glaubwürdiges Verzeichnis über das im Nachlass verbliebene Vermögen vorhanden, würde sich die Beklagte zufrieden geben (,waer sü zu frid“). Wäre das Nachlassvermögen bis zur Veröffentlichung des Geschäfts im Rat oder bis zur Aufnahme eines Inventars („Eröffnung des Geschaeffts vor [...] Rate oder bis $\beta$ [...] ain Inventarj gemacht") versiegelt geblieben, dann hätte sich feststellen lassen, was von dem Vermögen der Beklagten, und was davon dem Kind (und seiner Mutter) zugehörig gewesen sei. So aber habe Keck auch einiges von dem der Beklagten mit ihrem früheren Ehemann gemeinsamen Vermögen entfernt (,von ungetailtem Gut genommen"), und nun wisse man nicht mehr, was. Der Kläger sei daher mit Eigenmacht und somit unrechtmäßig (,,aus aignem Gewalt, nicht nach Ordnung der Rechten") vorgegangen; demnach wäre billigerweise er der Sanktion des Gesetzes "Si quis in tantam" (,, demnach er billich in [...] Straff") verfalle. Dr. Wepp räumt zwar ein, dass die Beklagte sich in der gleichen Weise wie der Bürgermeister Nachlassvermögen ihres früheren Mannes angeeignet habe, wenn aber der Kläger davor ein Inventar aufnehmen hätte lassen, dann wäre auch sie in derselben Weise vorgegangen (, also hiet sï im gevolgt"). Sie sehe sich nun aber nicht als dazu verpflichtet an, allein ein Inventar aufzunehmen.

Dem weiteren Einwand der Kläger, dass von Keck aus dem Nachlass von Vorchtenauer separierte Vermögen in gerichtlicher Sperre belassen worden sei (,sölh mit Gerichtshanden hiet lasse versperren“), hält die Beklagte entgegen, dass ihr dies nicht vom Gericht bekannt gemacht (, waer es ir [...] nit von Gerichts wegen verkhunt") worden sei, außerdem habe man diese Sachen zu einer Zeit weggebracht als sie lebensgefährlich erkrankt (,,in todtlicher Kranckhait“) war, weshalb sie bei diesem Vorgang selbst nicht anwesend sein konnte; sie habe aber auch niemanden anderen dazu delegieren können, weil alles so plötzlich („urbring“) gekommen sei.
In der Folge sucht Dr. Wepp auch die übrigen Einwendungen der Kläger zu entkräften. Zur heimlichen Öffnung des Testaments, welche Keck bestritten hat, meint er, die Beklagte wisse über das Geschäft nur, dass es vor den Rat gebracht worden sei. Es sei ihr aber nicht bekannt, wo Vorchtenauers etwa Siegel, Petschaft und Urkunden ("Insigill, Petschad und Brief“) verblieben sind. Der Bürgermeister habe ihr bloß ein paar Aufzeichnungen (,etlich klaine Zedln“) zugeschickt; auf dieser Grundlage hätte sie aber kein Inventar anlegen können. Was die vom Kläger aufgestellte Behauptung über die 900 Gulden Heiratsgut anbelangt, gesteht die Beklagte nun zwar ein, dass sie nicht gemeint habe, dieser Betrag wäre ihrem früheren Mann bar („,par Gelt") zugebracht worden, sondern es wären 300 Gulden als Heiratsgut (,,$z u$ Heimstewer") bloß versprochen gewesen; außerdem habe Vorchtenauer dafür 600 Gulden an Widerlegung und Morgengabe zugesagt.

Schließlich geht Dr. Wepp noch auf die von der Beklagten mit 4.000 Gulden bewerteten VeräuBerungen ihres früheren Gatten ein, welche weil sie ihr eigenes Vermögen beträfen (,weil es ihr Gut sei") - so wie der Anspruch auf das Heiratsgut zu behandeln seien, folglich gleichermaßen privilegiert (,mit zur Mitgift gehören und [...] geleich gefreit") wären. Sie wisse aber von niemandem, der aus diesen Verkäufen etwas schuldig geblieben sei, denn es stehen ihr weder Urkunden noch Verzeichnisse (,kainen Brief, [...] noch Register") ihres früheren Ehemannes (,irs Hauswirts") zur Verfügung. Die Frage, ob ihr Anspruch auf das Heiratsgut oder der des Kindes mehr privilegiert sei, sei rasch zu beantworten: "Dotis causa semper et ubique precipua est". ${ }^{59}$ Die Behauptung von Keck, dass er ihr nicht dazu geraten habe, das Vermögen ihres früheren

\footnotetext{
${ }^{59}$ Der Rückerstattungsanspruch auf die dos (Heiratsgut) war ein zentraler Leitsatz der Digesten (D. 23,3,70 = 50,17,85pr): STAGL, Favor dotis 1. Vgl. auch BRAUNEDER, Ehegüterrecht $140 \mathrm{ff}$.
} 
Ehemannes (,nicht wolt bekennen, daz er sÿ gehaissen hiet die Güter") aus dem Rathaus in ihr Haus zu bringen („in ir Hauß zu fuern“), wird von der Beklagten mit dem Hinweis entkräftet, dass er ihr dafür ja sogar seinen Wagen zur Verfügung gestellt habe. Die Behauptungen des Klägers seien daher unglaubwürdig (,unwirdig [...], im zu gelauben").

Die übrigen Behauptungen (,andern Artickl“) der Klägerseite, auf welche die Beklagte nicht ausdrücklich geantwortet (,nicht mit ausgedruckten Worten verantwurt") hat, werden von Dr. Wepp unter Verweis auf die schriftlichen Ausführungen („ir vorig Geschrift") - pauschal zurückgewiesen und nicht anerkannt (,vernaint und nicht bekennt").

Die Beklagte erklärt, sie sei den Klägern nichts schuldig und beantragt daher, die Klage zurückzuweisen (,begeret, daz sy [...] davon ledig erkennt [...] würde").

\section{E. Urteilsverkündung und Ausblick auf das weitere Verfahren}

Nachdem von den Parteien keine neuen Behauptungen mehr vorgebracht worden sind, erfolgt der Aktenschluss und es ergeht am 12. August 1507 die Entscheidung des Wiener Rats. "Mit Frag und Urtail" wird erkannt (,zu Recht gesprochen"): Paul Keck hat für das Klagebegehren weitere Beweise vorzulegen (,schuldig sein Clag, [...] zu weisen"); Dr. Wepp als Anwalt der Beklagten wird mit seinen Ansprüchen gegen den Kläger auf den Rechtsweg verwiesen ("möcht er in deshalben fürnemen wie Recht ist").

Der Prozess um Vorchtenauers Nachlass muss also fortgesetzt werden, wenn die Beklagte ihre Forderungen gegen den Bürgermeister durchsetzen will. Gegen die Entscheidung des Rats kann an den Landesfürsten "gedingt" werden.60

${ }^{60}$ WESENER, Einflüsse 57.
Die Entscheidung solcher "Gedinge"61 hat der Landesfürst zu dieser Zeit dem Hofgericht (dem königlichen Kammergericht) zu Wiener Neustadt überlassen ${ }^{62}$, um $\mathrm{zu}$ einem endgültigen Urteil zu gelangen. Die am 15. Jänner 1509 ausgefertigte Entscheidung des Wiener Neustädter Kammergerichts ${ }^{63}$ enthält aber kein Urteil in der Sache, sondern verpflichtet beide Parteien in Bezug auf den Nachlass ("gelassen Gut") von Stefan Vorchtenauer, über den jeweils in Besitz genommenen Anteil dieses Vermögens („souil ains yede Parthey deselben Guts eingenomen ond innhat") ein glaubwürdiges Verzeichnis, wie es sich gebührt und "Recht ist", aufzurichten. Beiden Streitteilen bleiben Einwendungen gegen das Verzeichnis der jeweils anderen Partei („ainen yeden Tail sein Einrede wider den andern“) vorbehalten. Danach solle der Rat die Entscheidung darüber treffen, "was Recht ist".

Bürgermeister Keck weigert sich - auf Grundlage seiner bisherigen Argumentation - weiterhin, das geforderte Vermögensverzeichnis vorzulegen und gibt bekannt, dass er inzwischen das von seinem Stiefsohn hinterlassene Haus "in der Lanndskron" für die Forderungen seines Mündels (wegen des bei Vorchtenauer „erlegten Gelts") gerichtlich beschlagnahmen habe lassen (, als des Puppil Underphant in Arrest durch die Obrigkait"). ${ }^{64}$

Die Beklagte bringt gegen die Erklärung des Klägers ebenfalls die bereits bekannten Argu-

${ }^{61}$ Von einer Appellation und einer Entscheidung des landesfürstlichen Gerichts als Instanz (wie im gemeinen Prozessrecht) kann wohl noch nicht gesprochen werden: Vgl. WESENER, Einflüsse 61f. mit weiteren Literaturnachweisen; vgl. WEITZEL, Appelation.

${ }^{62}$ Dazu WIESFLECKER, Österreich 251.

${ }^{63}$ Vgl. LAMPL, Haupturkunden, Nr. 6112: Der Hinweis auf den Nachtrag zu Bd. IV, 225, nach Nr. 5876 geht ins Leere, weil dieser Band nie erschienen ist. Den folgenden Ausführungen liegt der Text des Originals der Gerichtsurkunde: WStLA, HA-Urk. Nr. 1/1516 (fol. $1^{\mathrm{r}}$ ) zugrunde.

${ }^{64}$ LAMPL, Haupturkunden, Nr. 6112; vgl. AMEND, Arrest. 
mente vor, wonach dieser doch zur Inventarisierung des von ihm aus dem Nachlass ihres früheren Gatten übernommenen Vermögens verpflichtet sei und sprich in Zusammenhang mit der auf Anordnung des Klägers erfolgten Beschlagnahme des Hauses „in der Landskron“ von einer unrechtmäßigen Entziehung ihres Eigentums, sie sei vom Kläger "gewaltigclich entwert" worden. Dennoch willigt sie ein, dass ein Inventar über das von ihr aus dem Nachlass ihres früheren Mannes übernommene Vermögen aufgenommen wird - dies freilich unter Protest („,mit vorlaufender Protestacion“), weil sie dazu rechtlich eigentlich nicht verpflichtet sei65, und auch ohne damit anzuerkennen, dass es sich bei diesem Vermögen um "Vorchtenawer[s] aigenhafft Gueter" - und nicht um das von ihr in die Ehe eingebrachte Vermögen (,zugebracht aigenhafft Heyratgut auch Parafernalia").66

Die Inventarisierung dieses Vermögens erfolgt Anfang März 1509, und zwar im Haus „in der Landskron“, in Form eines durch Paul Müllauer, päpstlicher und kaiserlicher Notar67, vor Zeugen aufgenommenen notariellen Protokolls. ${ }^{68} \mathrm{Im}$ Anschluss daran wird von Mai 1509 bis gegen Ende 1510 in der Sache ${ }^{69}$ vor dem Rat weiter verhandelt. $^{70}$ Nach einer längeren Unterbre-

65 Ebd.

${ }^{66}$ WStLA, HA-Urk. Nr. 1/1516 (fol. 1). Zum Terminus Paraphernalia auch BRAUNEDER, Ehegüterrecht 140f.

${ }^{67} \mathrm{Er}$ ist (bis 1550) v.a. am Passauer Konsistorium in Wien nachweisbar. NESCHWARA, Notariat, 277, 283, 734.

${ }^{68}$ Der Verweis in LAMPL, Haupturkunden, Nr. 6112 (Anm. 8) auf den Nachtrag zu Band IV, 228 nach Nr. 5881 geht ins Leere, weil der 2. Teil zu Bd. IV nie erschienen ist. Die folgenden Ausführungen beruhen auf dem Original der Gerichtsurkunden in WStLA, HA, Urkunde Nr. 1/1516.

${ }^{69}$ LAMPL, Haupturkunden, Nr. 6112; vgl. VOLTELINI, 90.

${ }^{70}$ LAMPL, Haupturkunden, Nr. 6112 (372ff. Vorbringen der Beklagten und des Klägers Mitte Mai 1509; 374ff. Vorbringen der Beklagte in der 2. Jahreshälfte 1509; 376ff. Vorbringen des Klägers in der 1. Jahreshälfte 1510; 378ff. Vorbringen der Beklagten in der 2. chung wird der Prozess, nach dem (zwischen 1510 und 1514) erfolgten Tod des Klägers Paul Keck, 1516 wieder aufgenommen ${ }^{71}$ und kann nach mehrmaligem Schriftsatzwechsel Mitte September dieses Jahres mit Ratsurteil vorläufig beendet werden. ${ }^{72}$ Der Streit dreht sich, nachdem die Verpflichtung zur Inventarisierung zunächst weiterhin im Vordergrund der Auseinandersetzungen zwischen den Klägern und der Beklagten steht, seit 1510 vor allem um die Frage, ob der Beklagten zugestanden werden kann, dass sie über die von ihr behaupteten ehegüterrechtlichen Forderungen einen Würdigungseid (iuramentum in litem affectionis) leistet. ${ }^{73}$ Darüber führen nun beide Parteien, besser gesagt ihre Anwälte, das ganze Arsenal der Rechtswissenschaft gegeneinander ins Feld und bekämpfen sich mit Zitaten aus den Quellen und der Literatur des römischen und kanonischen Rechts; meist beziehen sie sich auf das Corpus Juris Civilis $^{74}$, und nur vereinzelt auf das Corpus Juris Canonici. ${ }^{75}$ Von den Belegen aus der Rechtsliteratur betrifft die Mehrzahl die Legisten, einige aber auch die Kanonisten: Die meisten Belege

Jahreshälfte 1510; 384ff. Vorbringen des Klägers noch vor Jahresende 1510).

${ }^{71}$ LAMPL, Haupturkunden, Nr 6112, Anm. 3, Rechtsnachfolger ist sein Sohn Dionys († um 1551/60), Ratsherr 1517/19, 1522/26, 1529, 1532/33, 1538/40, 1542/51, Raitherr 1519, Steuerherr 1539/40, Grundbuchsverwalter 1539, 1541/43 und Bürgermeister 1523/24, 1527, 1534/36: PERGER, Ratsbürger 176.

${ }^{72}$ LAMPL, Haupturkunden, Nr 6112 (386ff. Vorbringen der Klägerin; 391f. Urteilsverkündung).

${ }^{73}$ LAMPL, Haupturkunden, Nr 112 (377, 382f., 384f., 386).

${ }^{74}$ Der Codex wurde 16mal zitiert (ebd. 379, 380, 381, 382 383, 385, 386, 389, 390), die Digesten 14mal zitiert $(379,380,382,383,390)$; ferner wurde je einmal auf eine konkrete Kaiserkonstitution hingewiesen, nämlich auf einen "Text des Kaisers Diocletiani" (= C. 5,18,9) der mit Satz "Ingrediendi [enim] possessionem rerum dotalium" (385) bzw. auf das Gesetz, das mit den Worten "Si quis in tantam" (386) einleitet.

${ }^{75}$ Hierzu findet sich lediglich zwei Hinweise auf den Liber Extra (382). 
beziehen sich auf Baldus de Ubaldis (auch Baldaschini; 1344-1400) $)^{76}$, Bartolus († 1375) ${ }^{77}$, Bartholomäus de Salicieto (1363-1412) ${ }^{78}$, Jason de Mayno (1467-1519)79, Albericus de Rosciate († 1354; auch de Rosato genannt) ${ }^{80}$, Alexander de Imola († 1477; auch Tartagnus genannt), Raphael Fulgosius (1389-1427) ${ }^{81}$, Paulus Castrensis (auch de Castro genannt; † nach 1441) ${ }^{82}$, Cynus (Cino da Pistoia, um 1270-1336/7) ${ }^{83}$ und Angelus de Baldeschi (1328-1400). ${ }^{84}$ Andere Rechtsgelehrte (,Lerer der Recht" ${ }^{\prime \prime 5}$ ) werden vereinzelt zitiert. ${ }^{86}$ Das vor allem von Dr. Wepp forcierte Einwerfen von Argumenten auf Basis der gelehrten Rechte sowie das Zitieren der juristischen Literatur bringt der Beklagten auch den Vorwurf der Kläger ein, sie „understünd sich [...] [das Gericht] mit vil Scribenten der Recht [...] irr ze machen" - was von ihr mit der spöttischen Bemerkung an den Kläger quittiert wird, dass „sein[e] Advocaten [...] vielleicht der Rechten noch Schüler und unwissent seien".87 Die im letzten Prozessstadium ausgetauschten Schriftsätze sind aber geradezu als wissenschaftliche „Abhandlungen über den Schätzungseid und die Rechtsstellung der dos"88 zu werten.

\footnotetext{
${ }^{76}$ Sechsmal zitiert $(379,380,381,389)$.

77 Fünfmal zitiert $(382,383)$.

${ }^{78}$ Fünfmal zitiert $(379,381,382,382)$.

${ }^{79}$ Dreimal zitiert $(380,383)$.

${ }^{80}$ Dreimal zitiert $(381,383)$.

${ }^{81}$ Zweimal zitiert $(379,382)$.

82 Zweimal zitiert $(381,383)$.

${ }^{83}$ Zweimal zitiert $(381,383)$.

${ }^{84}$ Zweimal zitiert (383).

${ }^{85}$ Ebd. 379.

${ }^{86}$ Ebd. 380 Bartholomäus Socinus (um 1460-1507); 381 Henricus Bouhic († um 1350), Cardinalis (Hostiensis, auch Henricus de Segusia; † 1271), Anton de Butio († 1408), Johannes de Imola ( +1436$)$, Abbas Siculus († 1453/54; auch Nicolaus de Tudeschis oder Panormitanus genannt); 382 Philippus Franchus de Franchis (um 1450), Bartolus und seine Schüler („Bartolus et doctores"); 383 Johannes Faber (2. Hälfte 14. Jh.).

${ }^{87}$ LAMPL, Haupturkunden, Nr. 6112 (384, 381).

${ }^{88}$ Voltelini, Rezeption 90.
}

Das schließlich Mitte September 1516 geschöpfte Urteil $^{89}$ bringt wieder keine endgültige Entscheidung in der Sache: Es trägt den Klägern abermals die Errichtung eines Inventars über das von Paul Keck aus dem Rathaus weggebrachte Vermögen von Dr. Vorchtenauer auf, sowie den Nachweis, dass er dabei mit Wissen und Zustimmung der Obrigkeit sowie unter Beiziehung von glaubwürdigen Zeugen („guter Leut") vorgegangen ist. Die Beklagte muss die Höhe von Heiratsgut, Widerlage und des von ihr eingebrachten Paraphernalgutes sowie dessen Vermehrung durch Vermögensveräußerungen während der Ehe durch Vorchtenauer („,die Summa Gelt, [...] [die er] an irer stat emphangen $h a b^{\prime \prime}$ ) beweisen. ${ }^{90}$ Im Anschluss daran wird - zu einem nicht mehr feststellbaren Zeitpunkt - das landesfürstliche Regiment (Statthalter und Hofrat) der niederösterreichischen Länder ${ }^{91}$ angerufen. Dieses stellt im Jänner 1524 lapidar fest: Es habe keine der Parteien das "dargebracht und bewiesen", was ihnen mit Ratsurteil im September 1516 auferlegt worden ist. ${ }^{92}$ Die Beklagte wird von der Forderung der Kläger (,von der Clager Clag“) daher frei (,muessig und ledig“) gesprochen; der Tochter des Lukas Schnitzer bleibt freigestellt, über ihre Forderung einen Schiedsspruch zu erwirken. ${ }^{93}$

\footnotetext{
${ }^{89}$ LAMPL, Haupturkunden, Nr. 6112 (391f.).

${ }^{90}$ Ebd.

${ }^{91}$ WESENER, Einflüsse 57f.; vgl. WeITZEL, Berufung.

${ }^{92}$ LAMPL, Haupturkunden, Nr 6297a. (Verkündigung des Urteils durch den Landesfürsten Anfang März 1524).

${ }^{93}$ LAMPL, Haupturkunden, Nr. 6298a. Weitere Hinweise auf Fortlauf oder Ausgang des 1507 beim Wiener Stadtrat anhängig gemachten Rechtsstreits sind im Wiener Stadt- und Landesarchiv nicht mehr greifbar.
} 


\section{Der Streitgegenstand: Der Nachlass des Stadtschreibers Dr. Stefan Vorchtenauer}

\section{A. Allgemeines}

$\mathrm{Zu}$ Beginn der zweiten Phase des Prozesses Keck versus Wepp erfolgt im März 1509 tatsächlich die unter den Parteien strittige Inventarisierung des Nachlasses von Stadtschreiber Dr. Stefan Vorchtenauer.

Vorchtenauer ist der Sohn des Wiener Kürschners Lienhart Vorchtenauer. ${ }^{94}$ Nach der Wiederverehelichung seiner Mutter mit dem Wiener Bürgermeister Paul Keck wird er gelegentlich auch Stefan Keck genannt. ${ }^{95}$ Nach Absolvierung des Grundstudiums an der Artistenfakultät in Wien (1484 immatrikuliert, 1486 Baccalaureus artium, 1488 Magister artium) ${ }^{96}$ wird er an der Juristenfakultät immatrikuliert. ${ }^{97} 1495$ ist er als artium Doctor auch in der Juristenmatrikel von Padua nachweisbar. ${ }^{98}$ Vorchtenauer ist voll ausgebildeter Jurist. Als Stadtschreiber ${ }^{99}$ von Wien tritt er seit Ende 1499 in Erscheinung. ${ }^{100}$

Er hinterlässt eine ansehnliche juristische Fachbibliothek im Umfang von etwa 80, größtenteils handschriftlichen, und nur zum geringen Teil gedruckten Werken. Darunter finden sich die wichtigsten Quellen der Legistik und Kanonistik sowie eine Auslese der Postglossatoren- und Dekretalistenliteratur. ${ }^{101}$ Die Bücher und Hand-

${ }^{94}$ LAMPL, Haupturkunden, Nr. 5836a.

${ }^{95}$ AUW, MFJ II, fol. 33v .

${ }^{96}$ SZAIVERT, GALL, Matrikel 185 (1484 I A 4)

${ }^{97}$ AUW, MJF II, fol. 33 (1488 II).

98 UibleiN, Akten 699.

${ }^{99}$ Dazu PAuser, Scheutz, Frühneuzeitliche Stadt- und Marktschreiber 515, 518ff.

100 Dazu UHLIRZ, Quellen 48f. sowie PERGER, Ratsbürger 134ff., $138 f f ., 197$.

${ }^{101}$ Biographische Daten und Hinweise auf die Namen der im Folgenden vorgestellten Autoren und Bücher aus COING, Handbuch sowie SAVIGNY, Römisches Rechts im Mittelalter. schriften sind in diesem Inventar separat ausgewiesen, und enthalten auch mehr oder weniger genaue bibliographische Hinweise. Die überwiegend bloß fragmentarischen Angaben zu den angeführten Autoren sind in der Edition - so weit als möglich - ergänzt und mit biographischen Daten versehen worden.

Die Edition bezieht auch die übrigen Aufzeichnungen über die im Nachlass von Vorchtenauer befindlichen Gegenstände ein. Dadurch entsteht ein wirklichkeitsnahes Abbild der Alltagskultur im Haus eines der Führungsschicht angehörigen Wiener Stadtbürgers zu Beginn der Frühneuzeit. $^{102}$

102 Die frühneuhochdeutschen Bezeichnungen der einzelnen Gegenstände werden in einem Glossar (unten Anm. 166) in moderne Terminologie übersetzt bzw. erklärt. 


\section{B. Edition des Nachlassinventars ${ }^{103}$}

[fol.3 ${ }^{\mathrm{r}}$ Bibliothek:] ${ }^{104}$ Varia super prima et secunda ff veteris. Varia super secunda ff nouj. Varia super prima et secunda Inforciatj. ${ }^{105}$ Varia [fol. $3^{\mathrm{v}}$ ] super prima et secunda parte Codicis. Alexander106 super prima et secunda [parte] ff veteris. Alexander super prima et secunda [parte] ff novi. Alexander super prima et secunda [parte] Inforciatj. Alexander super prima [parte] Codicis. Abbas ${ }^{107}$ [Lectura aurea] super secundo [libris] decretalium. Abbas super tertio [libris] Decretalium. Abbas super quarto et quinto [libris] Decretalium. Repertorium Abbatis. ${ }^{108}$ Consilia Abbatis. Questiones Abbatis. Prima et secunda pars Speculatoris. ${ }^{109}$ Tertia et quarta pars Speculato-

${ }^{103} \mathrm{Im}$ Sinne der Empfehlungen des OECD-Global Science Forum (best practices for ensuring scientific integrity and preventing misconduct) habe ich darauf hinzuweisen, dass die Transkription dieses Inventars auf Grundlage der Original-Gerichtsurkunde (WStLA, HA-Urk. Nr. 1/1507, fol. $3^{\mathrm{r}}-4^{\mathrm{v}}$, [http:// vdu.uni-koeln.de:8181/mom/AT-WStLA/HAUrk/ 5852/charter] (abgerufen 9. 8. 2011) von meinem Mitarbeiter, Herrn Cand. iur. Nicolas Wimberger, hergestellt und von mir anschließend überarbeitet worden ist. Die Zuordnung der Buchtitel zum Bibliotheksverzeichnis sowie die Erklärungen im Glossar hat Herr Wimberger überwiegend selbstständig erarbeitet: Der besseren Lesbarkeit halber wurden die im Original vorhandenen Abkürzungen aufgelöst (die betreffenden Ergänzungen aber nicht ersichtlich gemacht).

${ }^{104} \mathrm{Zu}$ biographischen Daten und anderen Hinweisen auf die im Folgenden vorgestellten Autoren und Bücher siehe oben Anm. 101.

105 „ff" gibt die im Mittelalter gebräuchliche Abkürzung für die Digesten wieder; die Attribute "vetus", "novum" bzw. "inforciatum" entsprechen der Einteilung des auf 50 Bücher verteilten Digestenstoffes durch die Glossatoren: vetus $=1$. bis 24. Buch, 2. Titel; es folgt novum bis 38. Buch, letzter Titel; den Abschluss bildet infortiatum.

106 Alexander de Imola (auch Tartagnus, aus Imola; 1424-1477).

107 Abbas (Panormitanus, Nicolaus de Tudeschis; 13861445), Lectura aurea super quinque libris Decretalium.

108 Abbas (auch Panormitanus bzw. Nicolaus de Tudeschis).

${ }^{109}$ Guilelmus Duranti († 1296), Speculum iudiciale. ris. Paulus de Castro ${ }^{110}$ super prima et secunda [parte] ff veteris, super prima et secunda [parte] ff noui, super prima et secunda [parte] Codicis, super prima et secunda [parte] Inforciati. Repertorium Berchtarchini. ${ }^{111}$ Philippus Francus super sexto. ${ }^{112}$ Dini super regulis iuris. ${ }^{113}$ Singularia Ludowicj de Roma.114 Regule iuris Mercuriales ${ }^{115}$ Iohannis Anndree [! $]^{116}$ Repeticiones diverse. ${ }^{117}$ Baldus ${ }^{118}$ [Lectura] super Institutionibus et diversj tractatulj in vno volumine. Dictionarius [!] Albarici. ${ }^{119}$ Consilia Iohannis Caldrini. ${ }^{120}$ Consilia Angelj de Parusio. ${ }^{121}$ Iohannis de Annonia [?], ${ }^{122}$ Abbatis Lapi ${ }^{123}$ [in] vno volumine. Francisci de Zarabellis ${ }^{124}$ [!] [Lectura] super Clementinis. Baldus super Decretalibus. Jnstituta in antiqua impressura. ${ }^{125}$ Archidiaconus ${ }^{126}$ super decretalibus. Panormitanus ${ }^{127}$ super secundo formularium advocatorum. Decretum. ${ }^{128}$ Decretales [liber] Sextus cum Clementini. ${ }^{129}$ Liber impressus in pergameno Sextus

${ }^{110}$ Paulus de Castro (um 1400).

111 Johannes Bertachinus (auch Firmanis; 2. Häfte 15. Jh.), Repertorium iuris utriusque.

112 Philippus Francus († 1471), Super sexto libro Decretalium.

${ }^{113}$ Dinus de Mugello (de Rossonis; 1253-um 1300), Tractatus super regulis iuris.

${ }^{114}$ Ludovicus Romanus de Roma (auch Ludovicus Pontanus; 1409-1439).

${ }^{115}$ Quaestiones Mercuriales super regulis iuris.

${ }^{116}$ Johannes Andreae (1270-1348).

117 Repetitiones iuris canonici.

${ }^{118}$ Baldus de Ubaldis (um 1325-1400).

${ }^{119}$ Albericus de Rosate († 1354).

${ }^{120}$ Johannes Caldrinus (um 1500).

${ }^{121}$ Angelus de Ubaldis, Bruder des Baldus de Ubaldis.

122 Johannes de Annonia (aus Hainault = Hennegau/Belgien) [?].

${ }^{123}$ Abbas Lapus (auch Panormitanus bzw. Nicolaus de Tudeschis).

${ }^{124}$ Franciscus de Zabarellis (1360-1417).

${ }^{125}$ Institutionen.

${ }^{126}$ Archidiaconus (auch Guido de Baysio; 1250-1313).

${ }_{127}$ Abbas, auch Panormitanus bzw. Nicolaus de Tudeschis.

${ }^{128}$ Gratianus de Clusio (Mitte 12. Jh.).

${ }^{129}$ Clementinae, 1317 promulgiert Dekretalen von Clemens V. (Papst 1305-1314). 
Decretalium. ${ }^{130}$ Liber Sermonum scriptus cum diuidia parte impressura et adiuncto tractatulo scilicet de victoria Cristi et alio tractatulo Sancti Ysidori ${ }^{131}$ de summo bono in vno volumine. ${ }^{132}$ Liber scriptus cum titulo incipit prologus Machabeorum. ${ }^{133}$ Liber scriptus psalmorum. ${ }^{134}$ Liber scriptus in pergameno cuius titulus est summa Pisane. ${ }^{135}$ Liber scriptus per viam vocabularum declarans moralisaciones. ${ }^{136}$ Strabo $^{137}$ de situ orbis. Liber Horarum scriptus. 138 Officia Ciceronis. ${ }^{139}$ Liber scriptus in pergameno Iohannis de Monte Regio. ${ }^{140}$ Libri non ligati et impressi. ${ }^{141}$ Exemplare Iheronimj. ${ }^{142}$ Johannis Andreae super regulis iuris. ${ }^{143}$ Varia in tractatu super Tiberiadis. ${ }^{144}$ Cautele Bartholomei Cepelle de Verona. ${ }^{145}$ Iohannes Capoccj de dote et donatio propter nuptias. ${ }^{146}$ Ynstitucionum. ${ }^{147}$ Repeticiones in uno [!] septem quaternarum. Textus librorum Autenticorum cum vsibus feudo-

\footnotetext{
${ }^{130}$ Jeweils der Liber Sextus Decretalium, 1298 promulgiert von Bonifatius VIII. (Papst 1294-1303).

131 Isidorus Hispalensis (auch Isidorus von Sevilla; +636).

132 De summo bono et soliloquiorum.

${ }^{133}$ Liber Maccabaeorum (Buch des Alten Testaments).

${ }^{134}$ Liber Psalmorum (Buch des Alten Testaments).

${ }^{135}$ Bartholomäus Pisanus (auch de Rinonicis, von Pisa; um 1338-1401), Summa de casibus conscientiae, gedruckt Augsburg 1475.

${ }^{136}$ Wörterbuch über moralische Anwendungen.

${ }^{137}$ Strabon, antiker griechischer Geschichtssschreiber († um 25).

${ }^{138}$ Liturgisches Stundenbuch.

${ }^{139}$ Marcus Tullius Cicero (106-73), De officiis.

${ }^{140}$ Kunisperger bzw. Hans de Kungsberg, davon abgeleitet de Regiomonte (auch Johannes Müller, Johannes Francus oder Johannes Germanus genannt), Mathematiker, Mechaniker und Astronom (14361476).

${ }^{141}$ Ungedruckte und nicht gebundene Bücher.

${ }^{142}$ Sophronius Eusebius Hieronymus (347-420), einer der Kirchenväter.

${ }^{143}$ Johannes Andreae (1270-1348), Kanonist.

${ }^{144}$ Bartolus Tiberiadis, Tractatus de alluvione.

145 Bartholomaeus Caepolla († 1477), Tractatus de servitibus, cautelae, de simulatione contractuum, emptionum et venditionum.

146 Jakob von Viterbo (auch Capoccio; um 1255-1308),

Theologe.

${ }^{147}$ Institutionen.
}

rum. ${ }^{148}$ Instituta de Tortis. ${ }^{149}$ Totum Corpus Iuris Civilis. Practica Iohannis de Ferrario. ${ }^{150}$ Vocabularius iuris. $^{151}$ Innocencius super libris Decretoris [!]. ${ }^{152}$ Tractatus oblationum. ${ }^{153}$ Mamatrectus. ${ }^{154}$ Epithoma in Rhetoricam Ciceronis. ${ }^{155}$ Flos Testamentorum. ${ }^{156}$ Lanntzlotus ${ }^{157}$ super prima parte Codicis. Tractatus Ludouicj Polomonj. ${ }^{158}$ Tractatus vsurarum. ${ }^{159}$ Nicolaus ${ }^{160}$ super vltimis libris Codicis. Tractatus super titulo soluto Matrimonio.161 Tractatus de principibus. ${ }^{162}$ Speculum historiale Pogguis de Florentino. ${ }^{163}$ Tabula scripta cum certis ordinibus. Mappa mundi. ${ }^{164}$

${ }^{148}$ Authenticae und langobardisches Lehnrecht.

${ }^{149}$ Venezianischer Drucker (Ende 15. Jh.). Vgl. den Katalog der Frühdrucke der Universitätsbibkliothek Salzburg [http://www.ubs.sbg.ac.at/sosa/fr/frj.htm] (abgerufen 31.9. 2011), wo eine Ausgabe des Corpus Juris Civilis als "Instituta de tortis. Venedig, Baptista de Tortis. 1501" verzeichnet ist.

150 Johannes Ferrarius (auch Johannes Eisenmann; 1486-1558), Practica singularis ac perutilis.

${ }^{151}$ Rechtswörterbuch.

152 Sinibaldus Fliscus (auch Sinibaldo Fieschi; um 1195-1254), Innozenz IV. (Papst von 1243 bis 1254).

153 Marianus Socinus (1482-1556), Tractatus in materia oblationum.

${ }^{154}$ Alias Helias Helye (um 1400-1475), Drucker aus der Schweiz, Verleger des ersten Ausgabe des „Mammotrectus", eines religiösen Werks von Johannes Marchesinus (Ende 13./Anfang 14. Jh.).

155 Auszüge aus Ciceros (oben Anm. 139) rhetorischen Schriften.

${ }^{156}$ Rolandinus de Passagerius (13. Jh.). $\mathrm{Zu}$ ihm auch NESCHWARA, Notariat 20.

${ }^{157}$ Lancelottus Decius (1454-um 1535), Super prima parte Codicis.

158 Ludovicus Polomonus [?].

${ }^{159}$ Laurentius de Ridolfis (Ende 15. Jh.), Tractatus usuris.

${ }^{160}$ Johannes de Platea (2. Hälfte 15. Jh.), Super tribus ultimis libris Codicis, gedruckt in Lyon 1507 von Nicolaus de Benedictis. Vgl. dazu den Katalog der Frühdrucke der Universität Salzburg (Anm. 149).

${ }^{161}$ Ludovicus Pontanus (oben Anm. 114), De viro ff Soluto matrimonio.

162 Martinus de Garatis (15. Jh.).

163 Poggius de Florentino (auch Giovanni Francesco Poggio Bracciolini; 1380-1459).

164 Weltkarte. 
[Kleidung und Schmuck, Waffen] ${ }^{165}$ Ain klains Trühl, ain grosse Truhen mit ainem Fuess darinn, ain mandereine Schawben mit prawnen Attlas vberzogen, seinn die pessten Mäder der Frawen gewesen. Ain Swarze Tamaschkeme Schawben mit Vech vnnderfüetert. Ain graber Rokh mit Keelmader vnnderfüetert. Ain rote Häsukhen, ain grabe Häsukhen, ain graber Reitrokh mit weissem Thuoch vnnderfuetert, ain rote attlassene Ioppen, ain ausgenate zenndlene Ioppen. Ain prawne attlassene Ioppen, ain alter prawner Rokh, ain rotscharlach Parhosen, ain swarz alts Parhosen, ain alts rosinfarbs Parhosen, aber ain swarz Parhosen. Ain [fol.4 ${ }^{\mathrm{r}}$ grabe Kappen, ain lidrenes Bolier. Ain Tolich mit ainem silbrein Ortpannt, ain guldeiner Rin[n]g mit ainem grossen Turkhen, ain klains Ringl mit ainem Amatist, ain guldeinn Betschadt Rinng, ain guldein Rinng mit ainem Amatist, ain Rinngl mit ainem Rubindl, ain Silbrein Pismapfl, Zenndtstür, zway silbreine vbergolte Kneiffl, ain Scharlach gestulpt Paret, zway Par Credennzmesser. Ain Padtphaiten, vier Facillet, vier alt Ioppenphaiten, ain guldeine Hawben, vier Piret alt ond new, ain alter Reitrokh mit Fuchsrukh vnnderfuetert, ain alte Göpene, ain Par Sporn, ain Par Reitstifl, ain Reitswert, ain lannger Degen, ain Tollich mit ainem klain[n] silbrein Ortpanntl, ain Stükl Spenat, ain klains Stikl mit ainer herbein Leinwat, ain alt zenndleine Ioppen, ain alte swarze Hasugkhen, ain alter graber Huet, zwen alt Piberhuet.

[Küchengegenstände] Zingeschirr. Drey Achterinkandl mitsambt der Zaphmass, zwo Halbkanndl, vier klaine Kannndl Zapfmass, ain grosse plechene

\footnotetext{
165 Zur Erklärung der frühneuhochdeutschen Wörter wurden folgende Nachschlagewerke herangezogen: Heidelberger Akademie, Deutsches Rechtswörterbuch; GRIMM, GRIMM, Deutsches Wörterbuch; SCHMELLER, FROMMANN, Bayerisches Wörterbuch. Zur Erklärung topographischer Bezeichnungen CZEIKE, Groner-Wien-Lexikon. Dem sachverständigen Blick von Herrn Dr. Josef Pauser (Wien) ist es zu verdanken, dass in der Urkunde enthaltene undeutlich geschriebene Wörter in der Edition richtig wiedergegeben und auch im Glossar ihrer heutigen Bedeutung entsprechend erklärt sind.
}

Kandl, ain zinens Flaschl, ain zinene Halbflaschen. Zwen plechen Trachter. Drey zinenbeslagen Schüssel, ain Plat, vier Salssen-Schüssl. Zway küpfrene Schellel, ain klainer Kupferling, drew klaine messene Leuchterl, ain klains Glogkl, zwen Kuelkessl, ain Kuchlkessel, ain Mörser mitsambt dem Stössl. Zway Hakhmesser, vier hulzen Schusseln mitsambt ettlichen hulzen Teller.

[Waffen, Spiele] Ain Wezstain, ain klains Armbst, mit aim Hurnenpogen, ain klains Eyblein, ain klains Lädl mit etlichen Pfeylen, ain klains Stähl, zway lannge Messer, zwen Harnasch-Hanntschuech, ain pannzin Ioppen, ain Spilpret, ain Visier.

[Einrichtung, Getreide, Wein] Ain Plat auf ainn Tisch, mit Stiklein aufgeleimbt, vier gmalte Tuechl, ain Sesslstuel. Ain Raispetl, zwo Sidel fur ain Pett, ain grosse Sponnt mit aim gannzen Himl, darinn ain Vederpett, ain Tuchnat, ain gute, ain pöse Dekh, ain Hawbtpolster. Ain Kuss, ain klains slechts Petl, aber ain klains Sponpetl mit ainem halben Himel, aber ain klains gemalts Sponpettl, vier klain Spont mitsambt ainn klain Kindt-Spont, ain klaine Almar, ain grosser Spiegl, ain Scheerzewg, vier slecht Tisch, ain Truhen, funf Hawen, zway Khorst, zwo Klampfen, ain KandlRein, ain par Visch-Messer, ain halber Mutt Habern, allerlay Traid, Gerst, Maiz, Korn, ain Mutt, zwen Dreyling Weins, ain Halbfueder Weins, funf Dreyling Wein hat die Fraw gefechsent, drew alte Dreyling lare Vas, funf halb Dreyling lare Vas, vier klaine lare Anlegl, drey Putten.

[Bargeld, Forderungen] Item die fraw hat emphangen vierzigkh Phund Phen[n]ig aus des Burgermaister Geschaefft. Item nach seinem Tod ist gefunden worden sechs Phund Phennig. Hundert Reinladen, sonnst allerlay Laden per sechzigkh.

[Geräte, Werkzeug] Ain Haempotnig zu zehen Emern, aber ain alte Potnig bey aindlef Emern, aber ain alte Potnig bey zehen Emern, ain alte Speistruhen, vier Muelter, drew [fol.4v] Emerschaff, Zinntragen, Drew Viertl Schaeffl, ain Wasch-Schaff. Ain Vischwanndl, ain Hannzlpannkh, drew alte Tretscheffer, ain alter Schennkhstuel gannz zuprochen, ain Truhen zu ainen Tisch, newn Lesecheffl, ain alte Anricht, zwen alt Rost, drew alt Phannen, zwo alt 
Schauffl, ain klaine Saatruhen, Ain grosse Melmuelter, zwen Henngst auf ainn Herdt, zwo alt Weinlaitter, zwen alt Reidlin zu ain Kanerlir.

[Urkunden] Item in ainer verslossen Lad ist aufgebrochen worden, darinn drey Scatl seinn Missive ond annder Brief, seinn versecretirt worden, seinn auch in der vorbenannten Lad manigerlay Brief.

[Liegenschaften] Item von aufligunden Gueth der Kelberspewnt ligt bey Sand Vlrich, in der lanngen Maysen, vnd ist ain Iewch weniger ains Achtl, der Wayrring des ist ain Viertail. Item der Dubling des ist ain Viertail. Item der Newnperg ligt zu Salmonsdorff, ond ist drew Viertail. Item der Swikhart leyt zu Grinzing vnd ist sibenthalb Viertail. Item der Hainreichsbrun[ $n]$ ist ain tercial ains Iewchs, vnd ligt auch zu Grinzing. ${ }^{166}$

${ }^{166}$ Glossar (alphabetisch): Achterinkandl = Kanne $($ Mengenmaß $) ;$ Almar = Schrank; Amatist = Amethyst; Anlegl = Gefäß; Armbst = Armbrust; Betschadt-Rinng = Siegelring; Bolier = Hut; Credennzmesser $=$ Küchenmesser; Dekh, pöse = schadhafte Decke; Dreyling = Dreiling (Mengenmaß); Dubling = Döbling (Wien XIX. Bezirk); Emer = Eimer (Mengenmaß); Emerschaff = Eimerscheffel $($ Mengenmaß); Eyblein = Eibenbogen; Fartruhen = Schubkarren; Facillet $=$ Taschentuch; Frawen Closter Sannd Iacob = Jakobskloster (in Wien I. Bezirk); Fuchsrukh = Rückenfell des Fuchses; Fuess = Sockel; fechsen = ernten; Geschaefft $=$ Geschäft (letztwillige Verfügung); Glogkl = Glocke; Göpene = Joppe; Gueth, aufliguendes = Grundeigentum; Habern = Hafer; Haempotnig = Bottich (Mengenmaß); Hainreichsbrunn = Heinrichsbrunn (topographische Lage, Wien XIX. Bezirk); Hakhmesser = Hackmesser; Hannzlpannkh = Sitzbank; harnasch Hanntschuech = Panzerhandschuhe; Hasugkhen, Häsukhen = Überrock eines Mannes; Hawe = Axt; Henng[s]t = Vorrichtung zum Aufhängen von Küchengeräten; Herdt = Herd; $\operatorname{Him}[\mathrm{m}] l=$ Baldachin; hulzen = hölzern; Hurnenpogen $=$ Hornbogen; Iewch = Joch (Flächenmaß); Ioppenphaiten = Gehrock; Kandl = Kanne; Kanerlir = Rinne [?]; Keelmader = Fell vom Hals eines Maders (,Halsmarder“); Kelberspewnt = Kälberspeunt (topographische Lage, Wien, VII. Bezirk, bei St. Ulrich); Khorst = Getreidehohlmaß; Kindt-Spont $=$ Kinderbett; Klampfen $=$ Klammer; Kneiffl = Knopf; Kuchlkessl = Küchenkessel; Kuelkessl = Kühlkessel, Destilllierkessel; Kupferling = Kupfergefäß; Kuss = Kissen; Laden = Schrein, Truhe; lar = leer; Leinwat, herbein = Leinengewand; Lesecheffl = Weinle- sebutte; lidren = ledern; Mäder = Marderfell; manderein = aus Seide; Maysen, in der lanngen = in der langen Maisen (topographische Lage; in Wien, VII. Bezirk); Melmuelter = Mehlbehälter; messen = aus Messing; Missive = Briefe; Muelter = länglicher ausgehöhlter Behälter; $M u t t=$ Getreidemaß, -scheffel; Newnperg = Neunberg (topographische Lage, Wien, XIX. Bezirk); Ortpannt $[l]=$ Ortband; Padtphaiten = Badehemd; pannzin Ioppe $=$ Kettenhemd; Paret $=$ Barett; Pfeyle $=$ Pfeile; Phannen $=$ Pfannen; Piberhuet $=$ Hut aus Biberfell; Piret $=$ Barett; Pismapfl $=$ Kugel, Büchse in Apfelform mit Duftstoffen; Plat = Platte; plechen = aus Blech; Putte = Butte; Raispetl = Reisebett; Reidlin [Reutling] $=$ Stechmesser; Rein = Topf; Reinladen = Geldtruhe [?]; Rokh, graber $=$ grauer Rock; Rost $=$ Eisengitter; Rubindl = Rubin; Salmonsdorff = Salmannsdorf (topographische Lage; in Wien, XIX. Bezirk); Salssen Schüssl = Salzschüssel; Sand Vlrich = Ulrichskirche (in Wien, VII. Bezirk); Scatl = Schachtel; Schawben = Damenrock aus Seide; Scheerzewog = Scherzeug; Schellel = Schalen; Sidel $=$ Sitz(bank); Spenat = Leinwand; Spilpret $=$ Spielbrett; Sponnt = Bett; Stähl = Stahl; Stiklein = Stück; Suntag Reminiscere = zweiter Fastensonntag; Swikhart = Schwieghart (topographische Lage, Wien, XIX. Bezirk); tamaschkem = aus Damast; Tercial = Drittel; To[l]lich, Tollich = Dolch; Trachter $=$ Trichter; Traid $=$ Getreide; Tretscheffer $=$ Schaffel zum Einstampfen der Trauben mit den Füßen; Tuchnat = Bettzeug, Tuchent; Tuechl, gmalte = bemalte Tücher; Turkhe = Türkis; Vas = Fass; Vech = Fell $\left({ }_{\text {,Vieh }}{ }^{\prime \prime}\right)$; Vederpett = Federbett; versecretirt $=$ versiegelt; Visch-Messer $=$ Fischmesser; Vischwanndl = Fischwanne; Visier = Visier; Wayrring $=$ Währing (topographische Lage, Wien, XVIII. Bezirk); Wezstain = Schleifstein; Zaphmass = Zapfmaß; zenndlen = aus Seide; Zenndtstür [zenndlen stör]= Seidenstore; Zewgen = Zeugen; zinen = aus Zinn. 


\section{[Beurkundungsformular] ${ }^{167}$}

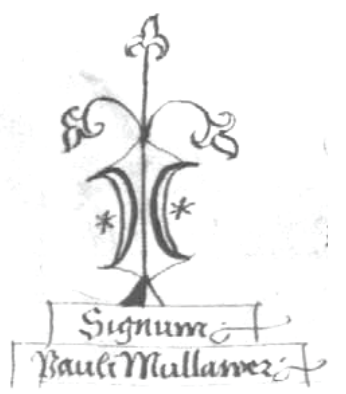

Beschehen zu Wienn in dem Hawss des obbemelten Doctor Hannsen Weppen an Freytag vor dem Suntag Reminiscere nach Cristi Geburdt, im funffenhundertisten ond newndten Iar, in Beywesen ond Gegenwurtigkait der ersamen Sebastian Pez, derzeit Hofmaister des Frawen Closter Sannd Iacob zu Wienn, Iacoben Weiss ond Micheln Rab, Mitburger daselbs als Zewgen zu Aufrichtung obbeschribens Inuentarien mitsambt mir obbemelten Paulln Mullauer Notarien eruordert ond sonnderlich gebeten.

167 Vgl. NesCHWARA, Notariat 25f.

\section{Literatur:}

Anja AMEND, Arrest, Arreststrafen, in: $\mathrm{HRG}^{2}, \mathrm{Bd} .1$ (Berlin 2008) 302-309.

Wilhelm BRAUNEDER, Die stillschweigenden Hypotheken und der österreichische Landsbrauch, in: Veröffentlichungen des Verbandes österreichischer Geschichtsvereine 19 (1972) 226-236.

Wilhelm BRAUNEDER, Die Entwicklung des Ehegüterrechts in Österreich. Ein Beitrag zur Dogmengeschichte und Rechtstatsachenforschung des Spätmittelalters und der Neuzeit (Salzburg 1973).

Wilhelm BRAUNEDER (Hg.), Juristen in Österreich 1200-1980 (Wien 1987).

Wilhelm BRAUNEDER, Gerhard JARITZ (Hgg.), Die Wiener Stadtbücher 1395-1430, Teil 1: 1395-1400 (=Fontes Rerum Austriacarum, 3. Abt.: Fontes Iuris, Bd. 10,1, Wien-Köln 1989).

Wilhelm BRAUNEDER, Widerlegung, in: HRG ${ }^{1}, \mathrm{Bd} . \mathrm{V}$ (Berlin 1998) 1346-1349.

Wilhelm BRAUNEDER, Eheliches Güterrecht, in: HRG², Bd. 1 (Berlin 2008) 1216-1221.

Wilhelm BRAUNEDER, Neuere Europäische Privatrechtsgeschichte (Skriptum Wien 172010).

Gerhard BUCHDA, Albrecht CORDES, Anwalt, in: $\mathrm{HRG}^{2}$, Bd. 1 (Berlin 2008) 255-263.

Helmut CoING, Handbuch der Quellen und Literatur der neueren europäischen Privatrechtsgeschichte, Bd. I: Mittelalter (München 1973).

Felix CZEIKE, Das große Groner-Wien-Lexikon (Wien 1974).

Heinrich Demelius (Hg.), Wiener Ratsurteile des Spätmittelalters ( $=$ Fontes Rerum Austriacarum 3. Abt.: Fontes Iuris, Bd. 6 Wien-Graz-Köln 1980).

Stephan DusIL, Grundpfandrechte, in: $\mathrm{HRG}^{2}, \mathrm{Bd} .2$ (Berlin 2010) 589-591.

Heidelberger Akademie der Wissenschaften (Hg.), Deutsches Rechtswörterbuch (Weimar 1914ff.).

Jacob GRIMM, Wilhelm GRIMM, Deutsches Wörterbuch, 33 Bde. (ND München 1984).

Paul HARRER-LuCIENFELD, Wien, seine Häuser, Menschen und Kultur, Bd. 1 (maschinschriftliches Manuskript im Wiener Stadt- und Landesarchiv, o.J.).

Josef LAMPL (Bearb.), Verzeichnis der Haupturkunden des städtischen Archivs 1494-1526 (= Quellen zur Geschichte der Stadt Wien, II. Abt.: Regesten aus dem Archiv der Stadt Wien, Bd. IV, Wien 19171919).

Gerhard JARITZ, Christian Neschwara (Hgg.), Die Wiener Stadtbücher 1395-1430, Teil 3: 1406-1411, (=Fontes Rerum Austriacarum, 3. Abt.: Fontes Iuris, Bd. 10,3, Wien-Köln-Weimar 2006). 
Christian NeschwarA, Anwalt, in: EdN, Bd. I (Stuttgart 2005) 483-485.

Christian NeschwARA, Geschichte des österr. Notariats, Bd. I: Vom Spätmittelalter bis 1850 (Wien 1996).

Christian NESCHWARA, Rechtsformen letztwilliger Verfügungen in den Wiener Stadtbüchern (13951430). Eine Bilanz aufgrund der vorliegenden Edition bis 1417, in: Thomas OleCHOWSKI, Christoph SCHMETTERER (Hgg.), Testamente aus der Habsburgermonarchie. Alltagskultur, Recht, Überlieferung (= BRGÖ 1; Wien 2011) 131-147.

Josef PAUSER, Martin SCHEUTZ, Frühneuzeitliche Stadtund Marktschreiber - ein Aufriss, in: Andrea GRIESEBENER u.a. (Hgg.), Stadt - Macht - Rat 1607. Die Ratsprotokolle von Perchtoldsdorf, Retz, Waidhofen/Ybbs, Zwettl im Kontext (St. Pölten 2008) 515-563.

Richard PERGER, Die Wiener Ratsbürger 1396-1626. Ein Handbuch (Wien 1988).

Friedrich Carl von SAVIGNY, Geschichte des römischen Rechts im Mittelalter, Bd. I-VI (Heidelberg 1815-1831).

Johann A. SchmelleR, KARL G. FrommanN, Bayerisches Wörterbuch, 2 Bde. (München ${ }^{2} 1872$ und

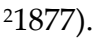

Jakob F. STAGL, Favor dotis. Die Privilegierung der Mitgift im System des römischen Rechts (WienKöln-Weimar 2009).

Willi SzAIVERT, Franz GALl (Bearb.), Die Matrikel der Universität Wien, Bd. II: 1451-1518, Text (GrazWien-Köln 1967).
Karl UHLIRZ, Quellen zur Geschichte der Stadt Wien, Abt. 2: Regesten aus dem Archiv der Stadt Wien, Bd. 1 (Wien 1898).

Paul UibleIN (Hg.), Akten der Theologischen Fakultät der Universität Wien (1396-1508), Bd. II (Wien 1978).

Hans von Voltelini, Zur Rezeption des gemeinen Rechts in Wien, in: Festschrift des akad. Vereines deutscher Historiker in Wien, hrsg. anläßlich der Feier des 25 jährigen Bestandes (Wien 1914), 79_ 129.

Jürgen WeITZEL, Appellation, in: $\mathrm{HRG}^{2}$, Bd. 1 (Berlin 2008) 268-279.

Jürgen WeitzeL, Berufung, in: HRG², Bd. 1 (Berlin 2008) $542 \mathrm{f}$.

Gunter WESENER, Einflüsse und Geltung des römischgemeinen Rechts in den altösterreichischen Ländern in der Neuzeit (16. bis 18. Jahrhundert) (Wien-Köln 1989).

Hermann WIESFLECKER, Österreich im Zeitalter Maximilians I. (Wien 1999).

Peter WRABETZ, Österreichs Rechtsanwälte (Wien 22008).

\section{Abkürzungen}

AUW, MJF II = Archiv der Universität Wien, Kodex J 2 (Matricula Facultatis Juristarum Studii Wiennensis, 2. Band: 1442-1557).

WStLA, HA-Urk = Wiener Stadt- und Landesarchiv, Hauptarchiv, Urkunden. 